\title{
Homozygous expression of the myofibrillar myopathy-associated p.W2710X filamin C variant reveals major pathomechanisms of sarcomeric lesion formation
}

Julia Schuld ${ }^{1}$, Zacharias Orfanos ${ }^{1,2}$, Frédéric Chevessier ${ }^{3,4}$, Britta Eggers ${ }^{5}$, Lorena Heil ${ }^{1}$, Julian Uszkoreit ${ }^{5}$, Andreas Unger ${ }^{6}$, Gregor Kirfel ${ }^{1}$, Peter F. M. van der Ven ${ }^{1}$ D, Katrin Marcus ${ }^{5}$, Wolfgang A. Linke ${ }^{6}$, Christoph S. Clemen ${ }^{3,7,8,9}$, Rolf Schröder ${ }^{3^{*}+}$ and Dieter O. Fürst ${ }^{1^{*}+}$

\begin{abstract}
Filamin C (FLNC) is mainly expressed in striated muscle cells where it localizes to Z-discs, myotendinous junctions and intercalated discs. Recent studies have revealed numerous mutations in the FLNC gene causing familial and sporadic myopathies and cardiomyopathies with marked clinical variability. The most frequent myopathic mutation, p.W2710X, which is associated with myofibrillar myopathy, deletes the carboxy-terminal 16 amino acids from FLNc and abolishes the dimerization property of Ig-like domain 24 . We previously characterized "knock-in" mice heterozygous for this mutation (p.W2711X), and have now investigated homozygous mice using protein and mRNA expression analyses, mass spectrometry, and extensive immunolocalization and ultrastructural studies. Although the latter mice display a relatively mild myopathy under normal conditions, our analyses identified major mechanisms causing the pathophysiology of this disease: in comparison to wildtype animals (i) the expression level of FLNc protein is drastically reduced; (ii) mutant FLNc is relocalized from Z-discs to particularly mechanically strained parts of muscle cells, i.e. myotendinous junctions and myofibrillar lesions; (iii) the number of lesions is greatly increased and these lesions lack Bcl2-associated athanogene 3 (BAG3) protein; (iv) the expression of heat shock protein beta-7 (HSPB7) is almost completely abolished. These findings indicate grave disturbances of BAG3-dependent and -independent autophagy pathways that are required for efficient lesion repair. In addition, our studies reveal general mechanisms of lesion formation and demonstrate that defective FLNc dimerization via its carboxy-terminal domain does not disturb assembly and basic function of myofibrils. An alternative, more amino-terminally located dimerization site might compensate for that loss. Since filamins function as stress sensors, our data further substantiate that FLNc is important for mechanosensing in the context of Z-disc stabilization and maintenance.
\end{abstract}

Keywords: Myofibrillar myopathy, Mouse model, Filamin, Myofibrillar lesions, Muscle damage, BAG3, HSPB7, Pathophysiology, Autophagy

\footnotetext{
*Correspondence: rolf.schroeder@uk-erlangen.de; dfuerst@uni-bonn.de

${ }^{\dagger}$ Dieter O. Fürst and Rolf Schröder are joint senior authors.

${ }^{3}$ Institute of Neuropathology, University Hospital Erlangen,

Friedrich-Alexander University Erlangen-Nürnberg, Schwabachanlage 6,

91054 Erlangen, Germany

${ }^{1}$ Institute for Cell Biology, Department of Molecular Cell Biology, University of

Bonn, Ulrich-Haberland-Str. 61a, 53121 Bonn, Germany

Full list of author information is available at the end of the article
}

C C The Author(s). 2020 Open Access This article is licensed under a Creative Commons Attribution 4.0 International License, which permits use, sharing, adaptation, distribution and reproduction in any medium or format, as long as you give appropriate credit to the original author(s) and the source, provide a link to the Creative Commons licence, and indicate if changes were made. The images or other third party material in this article are included in the article's Creative Commons licence, unless indicated otherwise in a credit line to the material. If material is not included in the article's Creative Commons licence and your intended use is not permitted by statutory regulation or exceeds the permitted use, you will need to obtain permission directly from the copyright holder. To view a copy of this licence, visit http://creativecommons.org/licenses/by/4.0/ The Creative Commons Public Domain Dedication waiver (http://creativecommons.org/publicdomain/zero/1.0/) applies to the data made available in this article, unless otherwise stated in a credit line to the data. 


\section{Introduction}

Heterozygous mutations in the human filamin $\mathrm{C}$ gene (FLNC) located on chromosome 7q32 cause hereditary and sporadic myopathies and cardiomyopathies with marked phenotypic variability [18, 23, 32, 86]. The first disease-causing FLNC mutations were described in the context of skeletal myopathies displaying the classical features of myofibrillar myopathies (MFMs; MIM\# 609524), which are morphologically characterized by sarcoplasmic protein aggregates and degenerative changes of the myofibrillar apparatus $[23,67,69,86]$. In addition, FLNC mutations were subsequently demonstrated to cause distal myopathies (MIM\# 614065) without evidence for protein aggregation [16, 26]. More recently, a growing number of truncating and missense FLNC mutations have been identified in cardiac diseases comprising restrictive, hypertrophic, dilated and arrhythmogenic cardiomyopathies (MIM\# 617047) [4, 5, 9, 18, 25, 56, 78]. A very recent extensive review provides a complete overview of the currently known approximately 325 FLNC variants. A large part of those are associated with skeletal myopathies and cardiomyopathies [83].

Filamin C (FLNc) is a large, dimeric, actin-crosslinking protein with a molecular mass of approximately $2 \times 290 \mathrm{kDa}$. It is predominantly expressed in crossstriated muscle cells, and localizes to myofibrillar Zdiscs, myotendinous junctions (MTJs), intercalated discs and the sarcolemma [80]. Its modular molecular architecture, consisting of an actin-binding domain followed by 24 immunoglobulin-like (Ig-like) domains, allows for multiple simultaneous protein-protein interactions, making FLNc a multi-adapter protein. Accordingly, more than 90 proteins have been identified as FLNc ligands, emphasizing its involvement in multiple scaffolding and signaling functions in muscle cells $[52,79,91]$. The essential role of FLNc for muscle development and maintenance is further highlighted by the effect of the abolition of its expression in mice and zebrafish. Loss of FLNc in mice causes a severe, perinatal lethal phenotype including defects in embryonic myogenesis resulting in a decreased number of primary fibers, excessive fiber size variation, and disturbance of sarcomere architecture [14]. In another mouse model, depletion of FLNc in the embryonic heart resulted in fetal death, while loss of FLNc in the hearts of adult mice caused severe dilated cardiomyopathy in most mice, leading to early death [92]. Corresponding studies in zebrafish demonstrated myofibril failure and altered sarcomeric architecture, but no effects on primary myogenesis [64].

In the present study, we explore the pathophysiology of the human FLNC p.W2710X mutation [ClinVar: NM_001458.4(FLNC):c.8130G > A (p.Trp2710Ter); Allele ID: 33353], which in the heterozygous state is the most frequently encountered human myopathy-causing
FLNC gene defect in various ethnic groups [37, 86]. It must be noted that another mutation mainly appearing in the population of Hong Kong [ClinVar: NM_ 001458.4(FLNC):c.8129G > A (p.Trp2710Ter); Allele ID: 682327] leads to the expression of an identical protein variant [44]. Both truncation mutations result in loss of the 16 carboxy-terminal amino acids, thus impeding the formation of dimers of Ig-like domain 24 of FLNc. As a result, the mutant protein has a strong tendency to aggregate and undergo proteolytic degradation $[47,86]$. Our previously reported heterozygous p.W2711X knockin mice, harboring the murine orthologue of the human FLNC p.W2710X mutation, represent the first patientmimicking genetic animal model for human filaminopathies [11]. These mice primarily demonstrated that heterozygous expression of this pathogenic FLNc variant results in myofibrillar instability with formation of FLNc- and Xin-positive sarcomeric lesions [11]. To explore the pathomechanism of this mutation in skeletal muscle more precisely, we now investigated mice homozygous for the FLNC p.W2711X mutation (Hom mice). These animals are viable and fertile, demonstrating that the exclusive expression of at least this particular mutant FLNc variant does not interfere with primary myogenesis. However, in comparison to wildtype (WT) and heterozygous animals [11], total FLNc levels were markedly reduced and concomitantly the protein was strikingly redistributed to areas of particular mechanic strain in skeletal muscle fibers. Moreover, these mice showed increased abundance of Z-disc streaming exemplified by the presence of sarcomeric microlesions (spanning up to five sarcomeres) and macrolesions (more than five sarcomeres and across multiple myofibrils), thus highlighting that FLNc might have a pivotal role for the structural and functional integrity of the myofibrillar apparatus. The dramatic decrease in the expression level of the autophagy-related small heat-shock protein HSPB7 in the muscle fibers of our Hom mice, and the absence of BAG3 from myofibrillar lesions in these mice suggest a cooperative role of these proteins in protection against, and repair of sarcomeric damage.

\section{Materials and methods \\ The FInc p.W2711X knock-in mouse model}

The Flnc c.8133G > A; p.W2711X knock-in mouse model B6J.B6-Flnc ${ }^{\text {tm1.1Rsdf }}$ (http://www.informatics.jax. org/allele/MGI:5907163) was generated as described before [11]. In brief, a point mutation in exon 48 of the Flnc gene leading to the insertion of a stop codon instead of a tryptophan residue was inserted by homologous recombination (GenOway, Lyon, France). The mutation was extensively validated by different methods. Genotyping was performed as described [11]. 
Mice were housed in isolated ventilated cages (IVC) equipped with spruce granulate embedding and a nest under specific and opportunistic pathogen-free (SOPF) conditions at $22 \pm 2{ }^{\circ} \mathrm{C}, 50$ to $70 \%$ air humidity, 70 air exchanges per $\mathrm{h}$, and a light-dark-cycle of $12 / 12 \mathrm{~h}$ with free access to water and food. Littermates were separated at weaning by sex and housed at maximal five animals per cage. Health monitoring was done as recommended by the Federation of European Laboratory Animal Science Associations (FELASA). Mice were handled in accordance with the German Animal Welfare Act and the German Regulation for the protection of animals used for experimental purposes or other scientific purposes. All investigations were approved by the governmental office for animal care (reference number 84-02.05.40.14.057).

\section{Histology, immunochemistry and light microscopy}

Soleus muscles from WT and mutant mice $(n=3-4$ per genotype) were snap frozen in liquid nitrogen cooled isopentane and stored at $-80^{\circ} \mathrm{C}$. To assess histology, $5 \mu \mathrm{m}$ thick transverse cryosections were stained with haematoxylin and eosin (HE) and modified Gömöri trichrome, and for cytochrome oxidase (COX) and succinate dehydrogenase (SDH) activity according to standard procedures.

For immunostaining, $10 \mu \mathrm{m}$ thick transverse or $6 \mu \mathrm{m}$ thick longitudinal cryosections were fixed with acetone $\left(-20^{\circ} \mathrm{C}\right)$ for $10 \mathrm{~min}$ or for $2 \mathrm{~min}$ with methanol $\left(-20^{\circ} \mathrm{C}\right)$ followed by $30 \mathrm{~s}$ acetone $\left(-20^{\circ} \mathrm{C}\right)$, respectively. After fixation, sections were rehydrated with phosphate-buffered saline (PBS) and incubated with blocking medium (10\% normal goat serum in PBS) for $45 \mathrm{~min}$ at $37^{\circ} \mathrm{C}$. Sections were incubated with primary antibodies diluted in $1 \%$ bovine serum albumin (BSA) in PBS overnight at $4{ }^{\circ} \mathrm{C}$. After washing with PBS containing $0.05 \%$ Tween 20 (PBST), sections were incubated at $37^{\circ} \mathrm{C}$ with the appropriate secondary antibodies diluted in 1\% BSA in PBS for at least $1 \mathrm{~h}$. Slides were rinsed in PBS and dipped in water before being mounted in Mowiol containing 0.25\% n-propyl gallate (Sigma P3130). Images were acquired using an LSM710 confocal laser scanning or a Cell Observer SD spinning disc microscope (Carl Zeiss $\mathrm{GmbH}$, Oberkochen, Germany).

\section{Infrared western blot analysis and protein quantification}

For comparative protein quantification, muscle samples from 6 to 10-month-old WT and mutant mice were dissected ( $n=4$ per genotype), weighed and snap frozen in liquid nitrogen. Samples were mechanically disrupted using a TissueLyser LT (Qiagen, Hilden, Germany) at $50 \mathrm{~Hz}$ and dissolved in $15 \mu \mathrm{l}$ urea buffer [ $2 \mathrm{M}$ thiourea, $7 \mathrm{M}$ urea, $5 \mathrm{mM}$ EDTA, $1 \mathrm{mM}$ DTT and protease inhibitors (Sigma, P8340) in $100 \mathrm{mM}$ Tris pH 8.6] per $1 \mathrm{mg}$ of muscle sample by homogenization for $3 \mathrm{~min}$ at $50 \mathrm{~Hz}$. Preheated SDS sample buffer was added to a final concentration of 2-fold and samples were incubated for $5 \mathrm{~min}$ at $55^{\circ} \mathrm{C}$. After quantitative analysis of a Coomassie-stained SDS-polyacrylamide gel, total protein concentration of the lysates was adjusted. For comparative quantitative blotting, identical total protein amounts were separated by SDS-polyacrylamide gel electrophoresis (SDS-PAGE) as previously described [42] and transferred onto a polyvinylidenfluoride (PVDF) membrane using a Transblot SD blot apparatus (BioRad, Munich, Germany). Membranes were incubated with Odyssey blocking buffer (LI-COR Biosciences, Bad Homburg, Germany) for $30 \mathrm{~min}$ at room temperature and incubated overnight at $4{ }^{\circ} \mathrm{C}$ with primary antibodies diluted in Tris-buffered saline with $0.05 \%$ Tween 20 (TBST). Subsequently, membranes were washed in TBST and incubated with IRDye-680 or IRDye-800-conjugated secondary antibodies (LI-COR Biosciences, Bad Homburg, Germany). Samples were analyzed using a LI-COR Odyssey Infrared Imaging System (LI-COR Biosciences). Integrated intensities of protein bands were quantified using the Odyssey Infrared Imaging Software v. 3.0 (LICOR Biosciences) and normalized to $\alpha$-tubulin or glyceraldehyde-3-phosphate dehydrogenase (GAPDH).

\section{Antibodies}

The following antibodies were used in this study: antiFLNc RR90, recognizing FLNa and FLNc [80], the rabbit sera FLNc d16-20 labelling Ig-like domains 16-20, and FLNC WT recognizing the carboxy-terminal 16 amino acids of Ig-like domain 24 of WT FLNc [11] were previously described. The mouse monoclonal antibodies (mAbs) XR1, labelling the isoforms XinA and XinB [82], T12 which recognizes a titin epitope close to the Z-disc [22], BB78 against myomesin [84], the rat mAb YL1/2 against tyrosinated $\alpha$-tubulin [88] and the rabbit serum detecting sarcomeric $\alpha$-actinin (RaA653) [80] have also been described previously. Antibodies recognizing type I, IIA IIX and IIB myosin heavy chain (MHC) isoforms (BA-F8, SC71, 6H1, BF-F3, respectively) and antidystrophin (MANDRA1) were obtained from Developmental Studies Hybridoma Bank (DSHB, Iowa City, USA). Anti-GAPDH (clone 5C6) was purchased from Calbiochem/Merck Millipore (Darmstadt, Germany), anti-vinculin (hVin-1, V9131) from Sigma Aldrich/ Merck, (Darmstadt, Germany), anti-slow MHC (NCLMHCs) from Novocastra/Leica Microsystems (Wetzlar, Germany), and anti-BAG3 and anti-HSPB7 from Proteintech, Manchester, UK. Secondary antibodies conjugated to Texas Red, Alexa Fluor 488, 594, 647, Cy2, Cy3 and Cy5 were from SouthernBiotech (Birmingham, AL, USA) or from Jackson ImmunoResearch (Ely, UK). For more detailed information on antibodies see Additional file 1: Table S1. 


\section{RNA extraction and real-time quantitative PCR}

To analyze Flna, Flnb and Flnc expression levels, RNA was isolated from the soleus muscles of 8-month-old WT and Hom animals using RNeasy Fibrous Tissue Mini Kit (Qiagen, Hilden, Germany) in combination with TissueLyser LT (Qiagen, Hilden, Germany). Genomic DNA was eliminated by RNase-free DNase I treatment. One microgram of total RNA was used for cDNA synthesis by the Omniscript RT Kit (Qiagen, Hilden, Germany) using random hexamer primers, according to the recommendations of the manufacturer. Real-time quantitative PCR reactions were performed using the SsoFast EvaGreen Supermix (BioRad, Munich, Germany) in a total reaction volume of $15 \mu \mathrm{l}$ and the CFX96 realtime PCR detection system (BioRad, Munich, Germany). For quantification, all reactions were performed in triplicates from 3 to 6 different cDNA samples prepared from different mice. The expression levels obtained were normalized to that of Gapdh and $\beta 2$ microglobulin (B2m). To determine the relative levels of mRNA expression between Hom and WT samples, ratios were calculated by the $\Delta \Delta C T$ method [46] using the Biorad CFX manager software version 3.1 and included a correction for amplification efficiency of the individual transcripts. Sequences of the applied oligonucleotides are provided in Additional file 1: Table S2.

\section{Electron microscopy}

Soleus muscle samples from 8-month-old WT and mutant mice ( $n=2$ per genotype) were immersed in Karnovsky fixative [33] containing $2.5 \%$ glutaraldehyde, $2 \%$ paraformaldehyde in $0.1 \mathrm{M}$ cacodylate buffer, $\mathrm{pH} 7.4$ for $2 \mathrm{~d}$ at $4{ }^{\circ} \mathrm{C}$. After washing twice with $0.1 \mathrm{M}$ cacodylate buffer, samples were trimmed to a smaller size and post-fixed in $1 \% \mathrm{OsO}_{4}$ for $1 \mathrm{~h}$ at room temperature. Samples were rinsed three times with double distilled water and counterstained with $4 \%$ uranyl acetate. Dehydration was carried out in a graded series of ethanol (50, 70, 95\% and twice in 100\% ethanol) for $15 \mathrm{~min}$ each followed by 3 consecutive steps of propylene oxide (Merck, Darmstadt, Germany). Samples were embedded in Epon resin (Serva, Heidelberg, Germany) which was allowed to polymerize for $48 \mathrm{~h}$ at $60^{\circ} \mathrm{C}$. To identify the specimens containing micro- and/or macrolesions, $1 \mu \mathrm{m}$ thick semithin sections were stained with toluidine blue and photographed (Axio Imager M1, Carl Zeiss, Jena, Germany). From these blocks $70 \mathrm{~nm}$ ultrathin sections were prepared (Ultracut S; Leica, Germany) that were placed on $2 \times 1 \mathrm{~mm}$ copper slot grids (Plano, Wetzlar, Germany) and examined with a Verios $460 \mathrm{~L}$ microscope (FEI - Thermo Fisher Scientific, Eindhoven, the Netherlands).

\section{Protein interaction and crosslinking assays}

A human FLNC cDNA fragment comprising Ig-like domains 15-23 was amplified with a full-length FLNC construct as template, and cloned into modified pAct2 and pLex vectors using standard protocols. Similarly, truncated variants of this fragment were cloned into the pLex vector. Transformation into L40 yeast cells, and culturing and testing for $\beta$-galactosidase activity was performed as described previously [81].

Protein cross-linking assays were performed essentially as described [29]. In brief, $\mathrm{His}_{6}$-tagged recombinant proteins were expressed in E.coli, purified using Ni-NTA agarose, and dialyzed against cross-link buffer (CLB; 600 $\mathrm{mM} \mathrm{NaCl}, 50 \mathrm{mM} \mathrm{NaP}$, $\mathrm{pH} 7.4,1 \mathrm{mM} \mathrm{MgCl}_{2}$, and 1 mM DTT). Subsequently, $50 \mu \mathrm{l}$ of a $10 \mu \mathrm{M}$ protein solution in CLB was equilibrated at $37^{\circ} \mathrm{C}$ for $10 \mathrm{~min}$, and $0.5 \mu \mathrm{l}$ of $65 \mathrm{mM}$ ethylene glycol bis (succinimidyl succinate) (EGS) in DMSO, or DMSO alone as a control, was added. The mixture was incubated for a further $10 \mathrm{~min}$ at $37^{\circ} \mathrm{C}$. After the reaction was stopped by addition of SDS sample buffer and incubation for $10 \mathrm{~min}$ at $95^{\circ} \mathrm{C}$, $5 \mu \mathrm{l}$ were analyzed by SDS-PAGE and western blotting using an antibody against the immunotag of the recombinant proteins.

Glutathione S-transferase (GST) pulldown assays were performed as described [51] using GST-tagged FLNc d18-21, and $\mathrm{His}_{6}{ }^{-}$and T7-tagged FLNc d18-21 and FLNc d15-17, respectively.

\section{Laser microdissection, sample processing and mass spectrometry}

The identification of different fiber types before laser microdissection (LMD) was enabled by staining for antislow MHC as described [89]. Non-fluorescent fibers were classified as type II fibers. A total of $250,000 \mu \mathrm{m}^{2}$ of the sarcoplasmic area of fibers was excised, collected and further treated for LMD as described [89], except that samples were reduced in $6.7 \mathrm{mM}$ dithiothreitol and alkylated with $5 \mathrm{mM}$ iodoacetamide. Approximately 200 ng peptides $(16 \mu \mathrm{l})$ of each sample was used for labelfree quantitative mass spectrometric (MS) analyses, performed on an UltiMate 3000 RSLC nano LC system (Dionex, Idstein, Germany) as described [49]. After each sample the column was equilibrated by a $1 \mathrm{~h}$ washing step. The HPLC system was online-coupled to the nano ESI source of a Q Exactive mass spectrometer (Thermo Fisher Scientific, Germany). In the ESI-MS/MS analysis, full MS spectra and MS/MS scans were performed as described [10] with the exception that only ions with a charge of +2 and +3 were selected for fragmentation.

\section{Data analysis of mass spectrometry}

Raw files were converted into mgf format using Proteome Discoverer 1.4 (Thermo Fisher Scientific, Germany) and then searched against a combined database containing the Swiss-Prot part of UniProt KB [76] for Mus musculus (version 2018_06, 16,985 entries) and the common 
Repository of Adventitious Proteins as contaminant database (version 2012.01.01, 115 entries). Shuffled decoy entries were generated for each protein using DecoyDatabaseBuilder [60]. For the identification of spectra, a respective concatenated target-decoy-database was used. Identifications were performed using Mascot 2.5 [Matrixscience Ltd., [59]], allowing 2 missed cleavages, precursor tolerance of $5 \mathrm{ppm}$ and fragment tolerance of $20 \mathrm{mmu}$. Carbamidomethylation (Cys) was set as static and carbamidomethylation (Asn), deamidation (Asn, Gln) and oxidation (Met) as variable modification. A spectral counting workflow was created in KNIME [version 3.5.3, [6]] to allow automated processing of sample analysis. The mass spectrometry proteomics data have been deposited to the ProteomeXchange Consortium via the PRIDE [58] partner repository with the dataset identifier PXD020097. Mascot dat files were used as starting point. First, the samples were processed by PIA $[73,77]$ to receive the peptide spectrum matches (PSMs) needed for spectral counting. The false discovery rate (FDR) was calculated for all files separately and an FDR of $1 \%$ on PSM level was maintained. The resulting PSMs were filtered for proteotypicity, i.e. only peptides, which were assigned to exactly one protein in the given database, were kept for further analyses. Here, the assignment to a contaminant was not considered to break the proteotypicity. The number of PSMs for each protein per run were normalized on all spectra per run, while also the non-proteotypic identifications were counted. These normalized counts are called spectral indices for each protein.

In order to ensure comparability of the data with previous experiments on myofibrillar myopathy patients, statistical analysis was performed as described [39, 48]. In brief: to identify significantly differentially expressed proteins, the ratio of the average spectral indices between phenotypes were calculated and Student's t-test was conducted on the spectral indices for each protein. Proteins with a $p$ value $<0.05$ were considered as significantly differentially expressed between the different phenotypes.

\section{General data analysis and figure preparation}

Data analysis and statistical evaluations were performed using GraphPad Prism version 7 for Windows (GraphPad Software, La Jolla, CA, USA) or Microsoft Excel 2016 in conjunction with the add-in "Real Statistics Resource Pack" version 5.5 by Charles Zaiontz, available at http://www.real-statistics.com (version 3.2.3). Data are given as median with IQR or as mean \pm SEM. $P$-values (Hom vs. WT) were calculated by Student's t-test, Kruskal-Wallis one-way analysis of variance and MannWhitney U (Wilcoxon rank-sum) test and quoted *< $0.05, *<0.01, \%<0.001$ and $* * 0.0001$ (precise choice of test given in Figure legends). Final assembly of the graphs and preparation of all figures was done using Corel Draw Graphics Suite X7 (Corel, Austin, TX, USA).

\section{Results \\ Filamin $\mathrm{C}$ is expressed at high levels in the soleus muscle of mice}

To determine which striated muscles express the highest FLNc levels, protein extracts from different skeletal muscles and the heart from WT mice were analyzed by western blotting. Quantification using the LI-COR Odyssey Infrared Imaging System demonstrated that the expression level of FLNc in the soleus muscle is significantly higher than in all other skeletal muscles, the diaphragm or the heart (Fig. 1a). For this reason, we decided to analyze the effects of the expression of only mutant FLNc in soleus muscle.

\section{Comparison of the expression levels of filamin C mRNA and protein in WT and Hom mice}

Reverse transcription quantitative polymerase chain reaction (RT-qPCR) to analyze Flnc mRNA expression revealed no significant differences between total Flnc mRNA levels in the soleus muscles of WT and Hom animals (Fig. 1b). Similarly, expression levels of Flna and Flnb mRNA were analyzed. Their expression levels were not significantly increased in soleus muscles of Hom mice (Fig. 1c).

We then analyzed the FLNc protein levels in our mice by western blotting, using two different antisera: one, directed against Ig-like d16-20, recognizes all FLNc, whereas the second one is directed against the 16 carboxy-terminal amino acids absent in the mutant and only reacts with the WT protein. This revealed that the total FLNc level in Hom mice was reduced to approximately $40 \%$ of that in WT mice (Fig. 1d, e). Staining with the second antiserum gave no signal in the Hom mice, confirming the complete absence of WT FLNc and the exclusive expression of mutant FLNc in those animals (Fig. 1d, e). We further confirmed this by proteomic analysis of the FLNc expression level in different fiber types of the soleus muscle of Hom and WT animals. In accordance with the data obtained by western blotting, in type I and type II fibers FLNc levels were reduced to 46.1 and $40.3 \%$ of the corresponding levels in WT muscle fibers, respectively (Fig. 2c).

Comparison of expression levels of proteins representing different sarcomeric sub-assemblies by quantitative western blotting did not reveal any significant differences in M-band, Z-disc, intermediate filament and Aband proteins or the muscle damage marker Xin (Fig. 2a, b) between WT and Hom mice. These data were grossly confirmed by proteomic analysis (Fig. 2c). By contrast, expression levels of the small heat shock protein HSPB7 (cvHsp, Hsp25-2) were hardly detectable in 


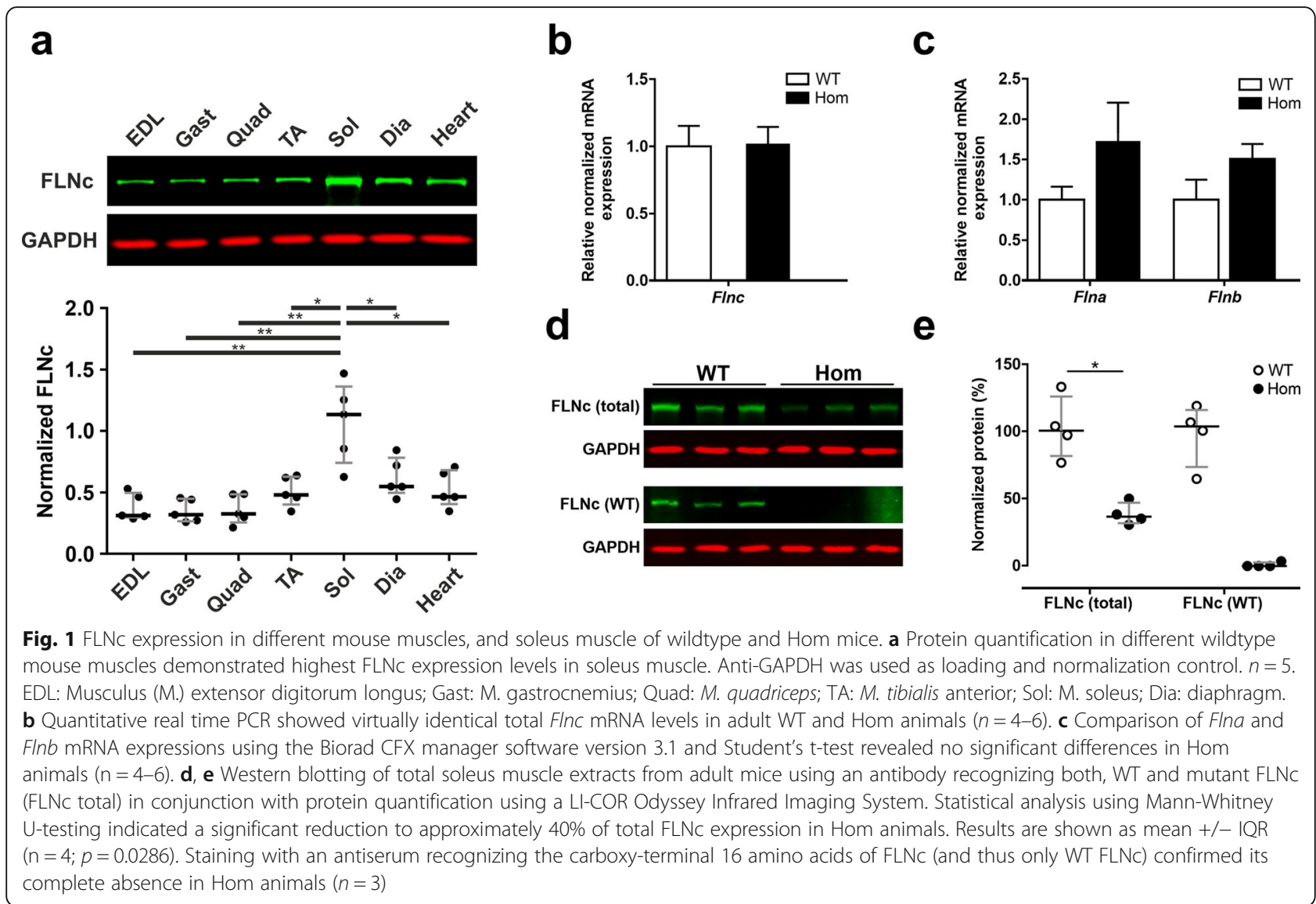

the muscle fibers of our Hom animals. Again, our western blot data were confirmed by proteomic analysis (Fig. 2ac). Notably, the latter method, based on MS analysis did not detect a single HSPB7 peptide in $90 \%$ of the samples acquired from type I or type II fibers derived from Hom mice. In addition, proteomic analysis indicated that FLNa and FLNb are neither expressed in the sarcoplasm of type I, nor of type II fibers (Fig. 2c). Protein levels of the chaperone-assisted selective autophagy (CASA)-related SYNPO2 (synaptopodin-2) protein were significantly increased in type II fibers of Hom mice, whereas type I fibers also showed a slight, but non-significant increase. Several other autophagy-related proteins were also increased in especially type II fibers (Fig. 2c).

\section{Homozygosity for the Flnc p.W2711X mutation causes a myopathic phenotype}

In transverse cryosections from soleus muscles of adult (8-10 months) and aged (20 months) WT and Hom mice (4 mice per genotype) stained with haematoxylin and eosin (HE; Fig. 3a), we investigated the number of fibers with centrally located nuclei. Numbers were statistically evaluated using a chi-squared test and demonstrated an increase in the number of centrally located nuclei in adult Hom animals (Hom: $4.40 \pm 0.52$ vs WT: $1.28 \pm 0.44, n=3$, $p=0.0033)$ and even more in aged Hom animals (Hom: $9.13 \pm 1.16, n=4$ vs WT: $1.1 \pm 0.40, n=3, p<0.0001$, Fig. 3b; Kruskal-Wallis test, Chi-square). Soleus muscles of Hom animals showed a slight increase of endomysial connective tissue, a rounding of muscle fibers and multiple atrophic muscle fibers. In addition, Hom soleus muscles contained degenerating fibers, which were never observed in WT muscles (Fig. 3a). Moreover, in nearly all soleus muscle samples the conspicuous presence of areas with increased fibrosis sometimes resembling myotendinous junctions was noted (Fig. 3a, asterisk). Staining with modified Gömöri trichrome, SDH and COX showed no evidence of mitochondrial abnormalities in Hom animals (Additional file 1: Figure S1).

To investigate the effect of mutant FLNc expression on fiber type and fiber size distribution in Hom animals, cryosections of soleus muscles were analyzed for expression of fiber type specific MHC, followed by statistical evaluation (Supp. Figure S2A-C). This revealed that fiber type composition is not significantly different in the soleus muscles of Hom mice compared to WT mice (Additional file 1: Figure S2B,C). Moreover, fiber number and fiber size (minimal diameter) were analyzed in cryosections stained for dystrophin. Adult and aged Hom soleus muscles contained significantly fewer fibers (Mann-Whitney 


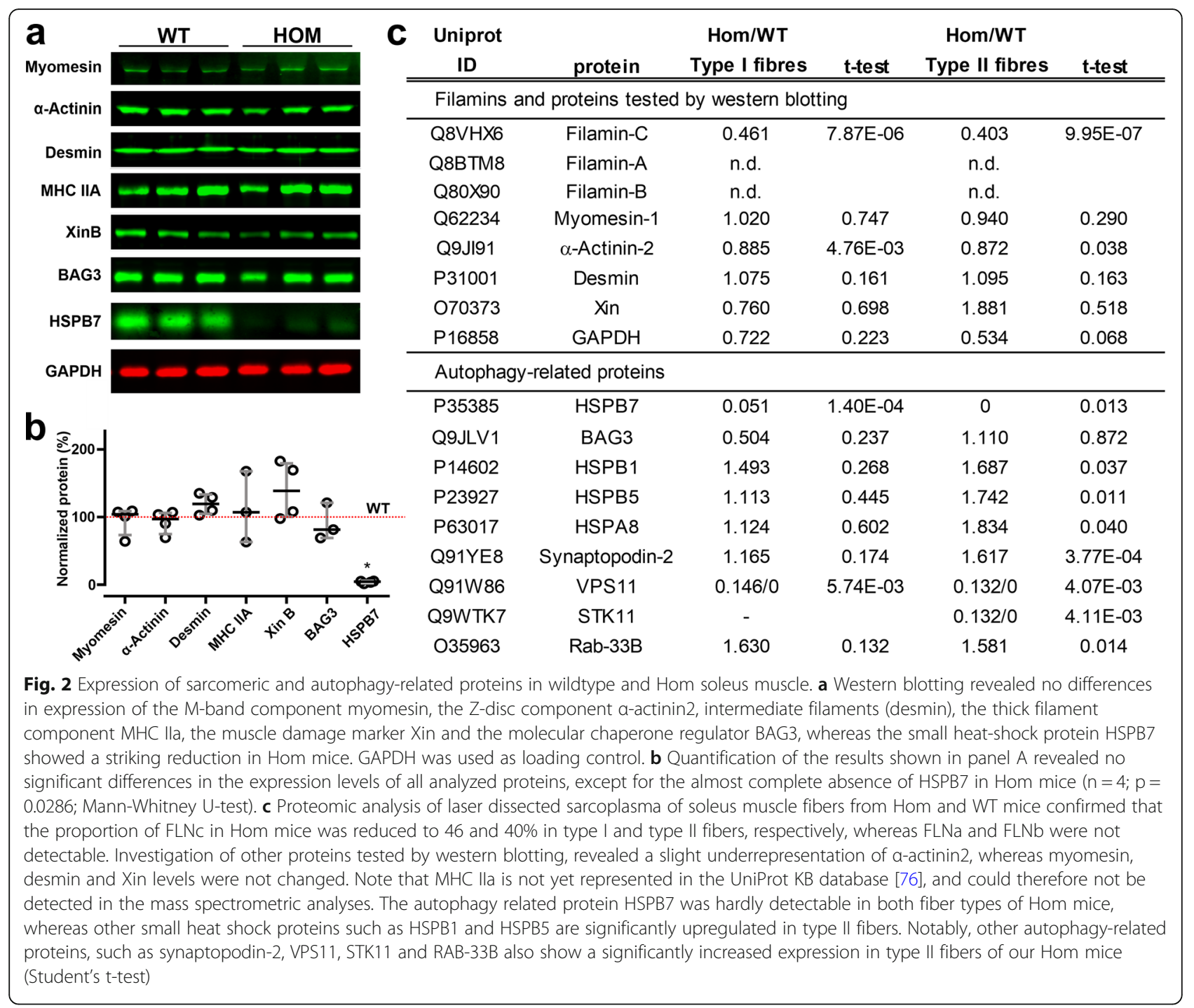

U-test, 6 mice per genotype; adult: WT: median 795 fibers, IQR 266 vs Hom: 614 fibers, IQR 245, $p=0.0152$; aged: WT: median 858 fibers, IQR 75 vs Hom: 596 fibers, IQR 155, $p=0.0043$ ) (Fig. 3c), although soleus muscle weight compared to body weight was not decreased. This lack of fibers was compensated by a significantly increased fiber size for MHC I-positive fibers in adult Hom mice (Kruskal-Wallis with post-hoc analysis using the MannWhitney U-test) 3 mice per genotype; WT: $31.3 \mu \mathrm{m} ; 275$ fibers, IQR 7.4 vs Hom: $34.8 \mu \mathrm{m} ; 216$ fibers, IQR 9.2, $p<$ $0.0001)$ as well as MHC IIA-positive fibers in both, adult and aged Hom mice (3 mice per genotype; adult: WT: $28.3 \mu \mathrm{m}$; 623 fibers, IQR 5.0 vs Hom: $31.4 \mu \mathrm{m}$; 422 fibers, IQR 6.0; 3 mice per genotype, $p<0.0001$; aged: WT: $34.7 \mu \mathrm{m} ; 560$ fibers IQR 5.0 vs Hom: $35.6 \mu \mathrm{m}$; 326 fibers, IQR 7.6;, $p=0.0061$ ) (Fig. 3d-g). By contrast, the size of MHC I-positive fibers in aged mice (3 mice per genotype), was significantly reduced in Hom mice: WT: $36.5 \mu \mathrm{m} ; 407$ fibers, IQR 5.5 vs Hom: $34.1 \mu \mathrm{m}$; 452 fibers, IQR 8.4; $p<$ 0.0001. (Fig. 3d-g).

\section{Reduced expression and altered distribution of mutant filamin $\mathrm{C}$ in Hom mice}

Cross sections of soleus muscles were analyzed for localization of FLNc and the muscle damage marker Xin. Whereas in the muscle fibers of WT animals FLNc was homogeneously expressed in all muscle fibers, soleus muscles from Hom animals only showed minimal staining (Fig. 4a). Instead, brightly stained foci were observed that also contained Xin, indicating that these structures represented muscle damage areas. Analysis of the Xin-positive area compared to the complete area of muscle fibers indicated that Hom fibers displayed significantly more muscle damage (median of lesion area in \% of WT: 0.48 vs Hom: 1.97; $p=0.0022 ; 6$ mice per genotype; Mann-Whitney Utest Fig. 4b). Staining of longitudinal sections unequivocally 

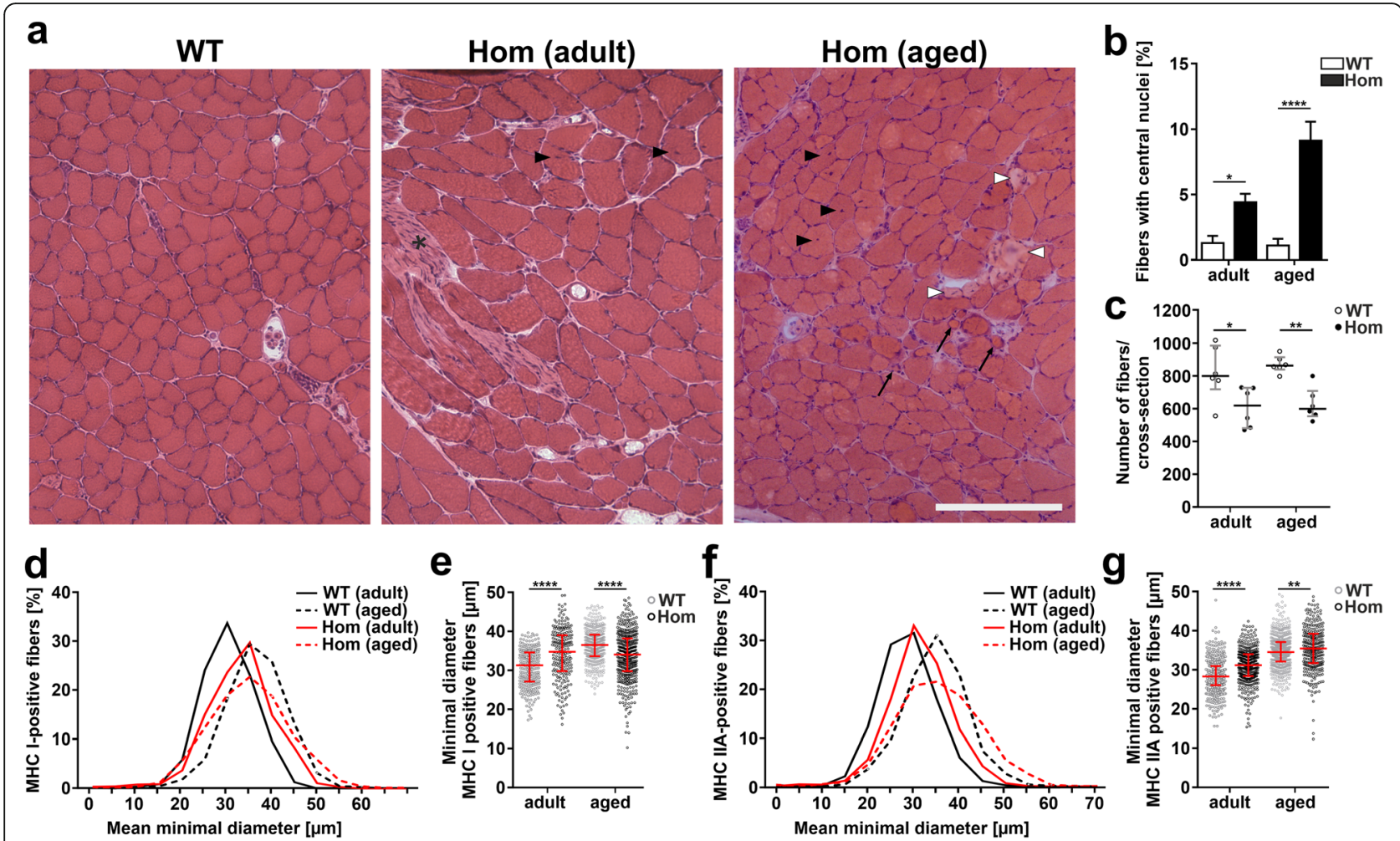

Fig. 3 Histological staining and analysis of fiber size, fiber number and fiber type in M. soleus. a Hematoxylin and eosin-stained transverse cryosections of WT and Hom mice. The sections of the Hom mice show increased fibrosis (asterisk), several fibers with central nuclei (black arrowheads), and very small fibers (arrows) and degenerating fibers in the aged Hom mice (white arrowheads), indicating an increased myopathic phenotype in these animals. $\mathbf{b}$ Statistical evaluation of respective percentages of fibers with centrally located nuclei using Kruskal-Wallis test and chi-squared independence tests. The number of muscle fibers with centrally located nuclei is significantly increased in adult and aged Hom animals (adult: $n=6 ; p=0.0033$; aged: $n=6, p<0.0001$ ). $\mathbf{c}$ The total number of fibers is significantly lower in the soleus muscle of Hom animals (adult: $n=6 ; p=0.0152$; aged: $n=6, p=0.0317$ ). $\mathbf{d}$-e When compared to WT mice, the mean minimal diameter of type I fibers is significantly larger in adult Hom animals $\left(^{* * *} p<0.0001\right)$, whereas they are smaller in aged mice $\left.{ }^{* * * *} p<0.0001\right)$, indicating that in Hom mice type I fibers don't increase their size as much as in WT animals during aging. $\mathbf{f}-\mathbf{g}$ When compared to WT mice, the mean minimal diameter of type II fibers is significantly larger in adult and aged Hom animals (***p $<0.0001,{ }^{* *} p=0.0061$ )

identified these intensely stained structures as sarcomeric micro- and macrolesions, rather than amorphous aggregates (Fig. 4c). Whereas in WT soleus muscles large quantities of FLNc were localized to Z-discs, in Hom muscle fibers staining of Z-discs was far less intense. Instead, FLNc was detected mainly in lesions and MTJs (arrowheads in Fig. 4c; Additional file 1: Figure S3). The signal intensity of FLNc localized to these structures was compared to that of titin using the Zen software line profile tool of the confocal microscope (Fig. 4d) and by comparison of the mean FLNc grey value normalized to the titin grey value in individual Z-discs (Fig. 4e). Statistical analysis using Kruskal-Wallisand subsequent Mann-Whitney U-test revealed a highly significant reduction of signal intensities of FLNc in the Zdiscs of Hom soleus muscle fibers (Fig. 4e).

\section{Distribution of titin and filamin $\mathrm{C}$ in macrolesions}

Previously, we identified FLNc and Xin as major components of micro- and macrolesions, that are supposed to protect myofibrils against further damage [43, 55].
Analysis of our adult Hom animals revealed high levels of FLNc in these lesions (Fig. 4a, b, Fig. 5a) that showed non-homogeneous FLNc signal intensities (Fig. 5b, c). Co-staining for a Z-disc epitope of titin (Figure 5B,C) combined with a line profile showed that areas with reduced FLNc signals precisely overlap with Z-discs. The distance between Z-disc titin signals within the lesions was approximately $7.2 \mu \mathrm{m}$, while in normal appearing sarcomeres the spacing was $2-2.5 \mu \mathrm{m}$.

The increased staining for FLNc in MTJs of Hom animals (Fig. 4c), prompted us to directly compare signal intensities of FLNc in these structures. Double staining with an antibody against vinculin to identify MTJs, showed a more intense staining in Hom mice, indicating strong accumulation of mutant FLNc in the MTJs of these mice (Additional file 1: Figure S3).

\section{Sarcomeric pathology at the ultrastructural level}

Light microscopy of toluidine blue stained semi thin sections from Epon embedded soleus muscle of adult Hom 
a
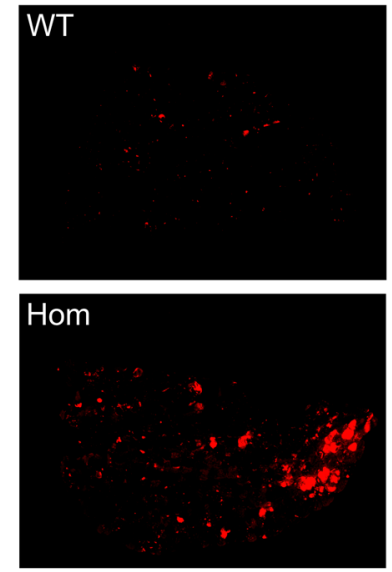

b

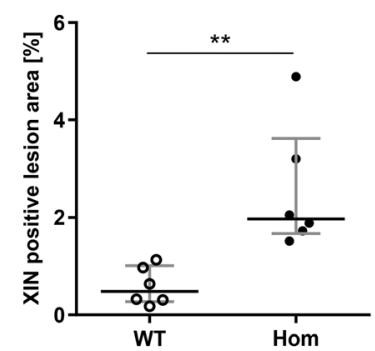

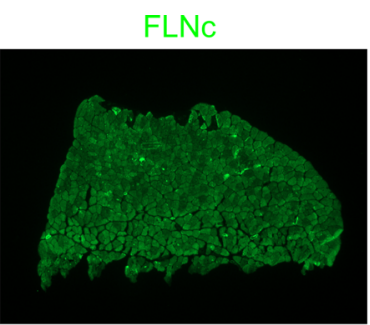
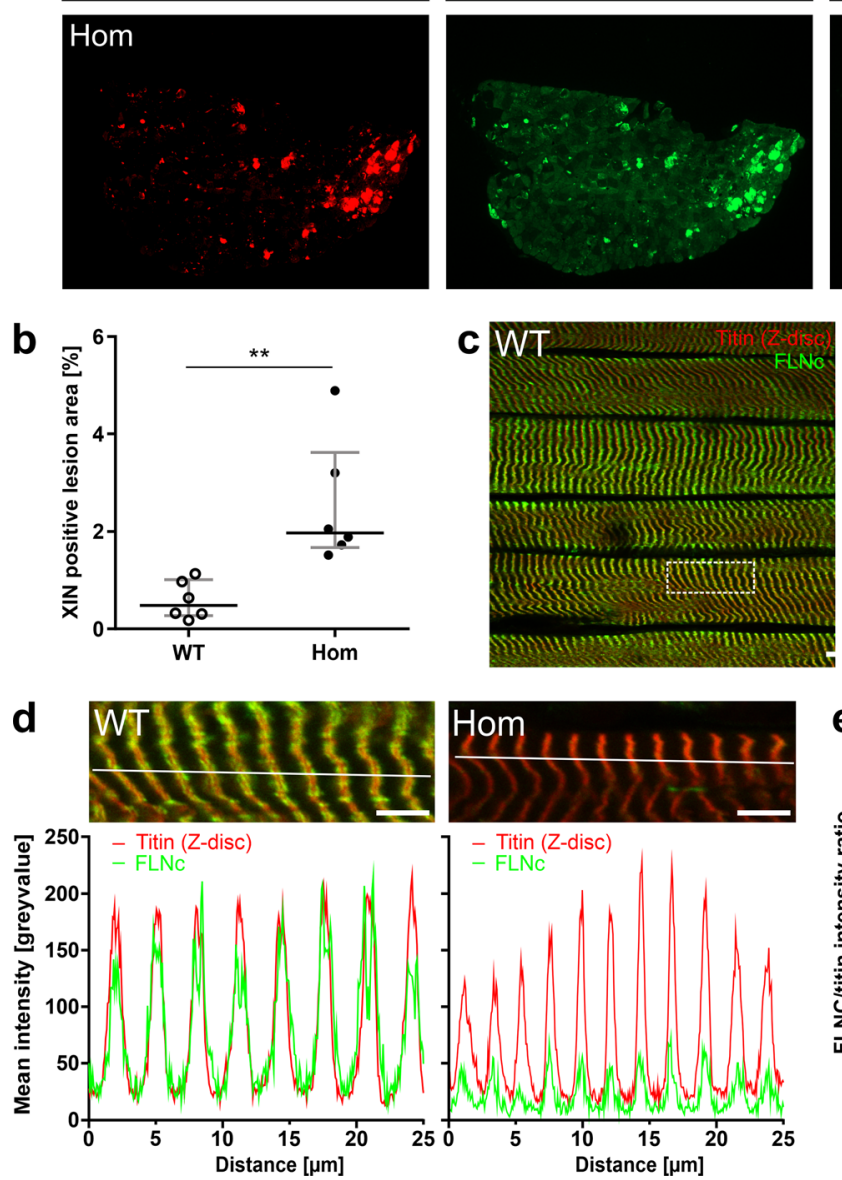
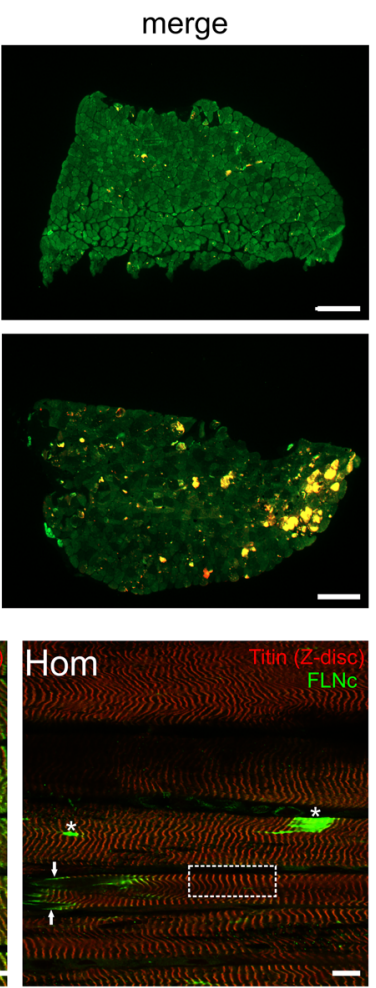

e

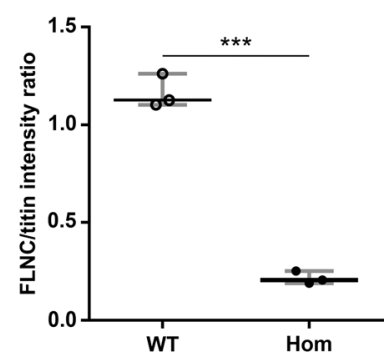

Fig. 4 Muscle damage and FLNc content in soleus muscles of adult WT and Hom mice. a Immunostaining of soleus muscle cross sections for muscle damage marker Xin reveals increased muscle damage in Hom animals. FLNc colocalizes with Xin in damage areas. Note that identical scan settings were applied. $\mathbf{b}$ Statistical analysis of the area of muscle damage using the Mann-Whitney U-test reveals significantly increased damage in Hom mice: median of lesion area in \% of total muscle area, 0.48 in WT versus 1.97 ( $n=6$ in each group; $p=0.0022$ ). $\mathbf{c}$-e Comparison of FLNc and titin expression levels in soleus muscle of wildtype and Hom mice. c Double staining of longitudinal sections of soleus muscle for FLNc and an epitope of titin close to the Z-disc shows significantly reduced levels of FLNc in Z-discs of Hom mice. Note that identical scan settings were used. Instead, FLNc was found highly concentrated in lesions (asterisks) and MTJs (arrows). d Illustration of Zen software line profiles of the mean immunofluorescent intensities of FLNc and titin in Z-discs of WT and Hom mice using the line profile option of the microscope and Zen 2 software Version 2.0.0.0. Note comparable intensities of FLNc and titin signals in Z-discs of wildtype mice but reduced FLNc intensity in Hom mice. e Quantification of FLNc Z-disc signal corrected against the background and normalized against titin confirms a highly significant reduction of FLNc levels in Z-discs in Hom mice. Statistical analysis was performed for sections from 3 mice of each genotype and in each 10 Zdiscs were included in the calculations. Kruskal-Wallis test one-way analysis of variance; post-hoc analyses by performing Mann-Whitney U-tests of both groups (median of WT: 1.16, median of Hom: 0.22; $p<0.0001$ ). For all experiments the RR90 antibody was used to detect FLNc. Bars: $200 \mu \mathrm{m}$ $(\mathbf{a}), 10 \mu \mathrm{m}(\mathbf{c}), 5 \mu \mathrm{m}(\mathbf{d})$

mice revealed extended dark appearing regions lacking the typical cross striated pattern of the surrounding myofibrils (Fig. 6a). Ultrathin sections of specimens containing such regions with a perfect longitudinal orientation were analyzed by transmission electron microscopy. This confirmed the presence of sarcomeric macrolesions, and showed that they always start at one Z-disc and end at another Z-disc (Fig. 6b). Within 

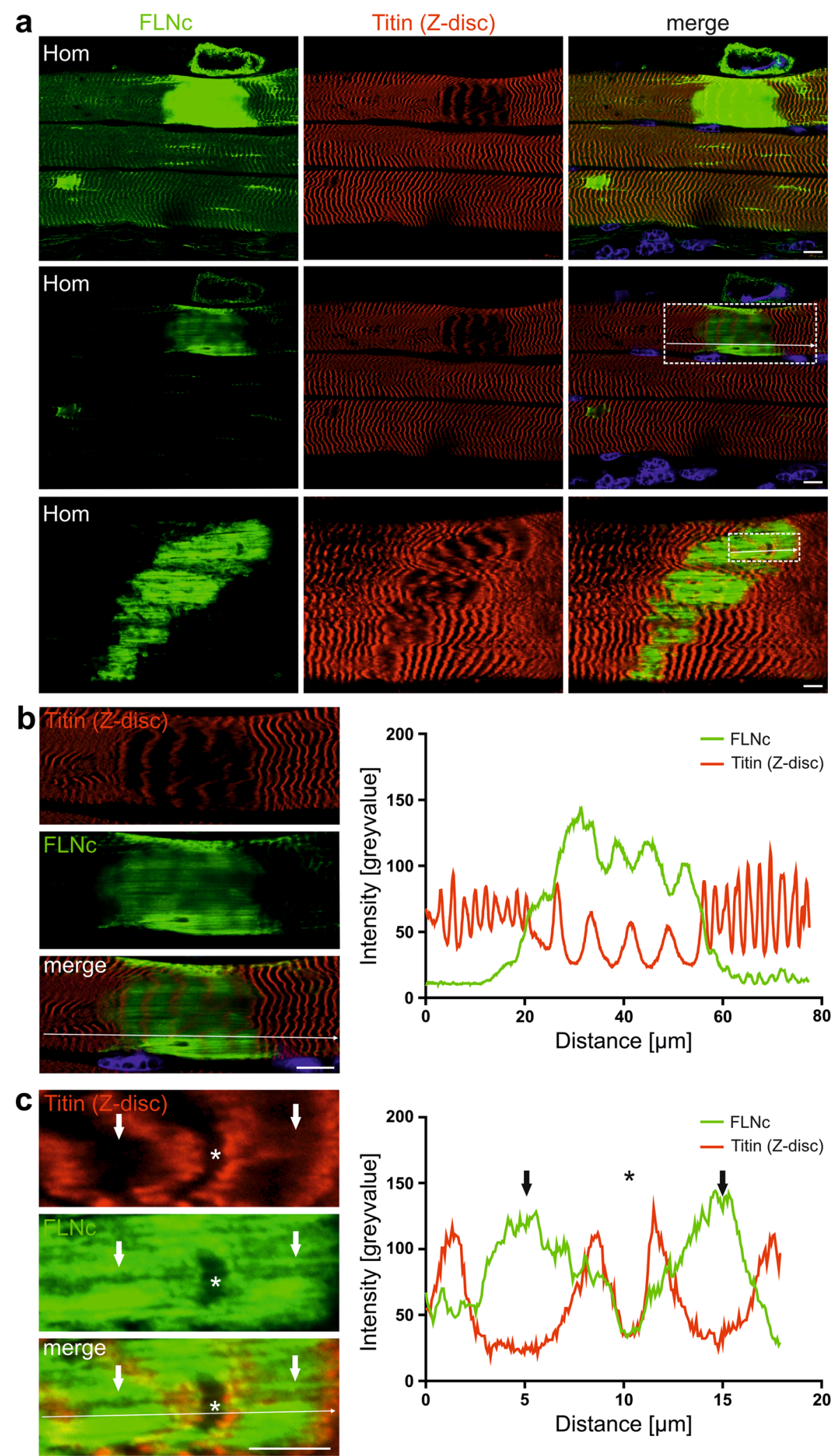

Fig. 5 (See legend on next page.) 
(See figure on previous page.)

Fig. 5 Distribution of FLNc and titin in myofibrillar lesions. a Longitudinal cryosections of soleus muscle from an adult Hom mouse stained for FLNc and titin. The upper panel shows an image with increased gain settings necessary to reveal localization of mutant FLNc in Z-discs. Macroand microlesions are strongly stained. The middle panel shows the identical part of the section with a gain setting enabling localization of FLNc within lesions. The lower panel shows a second fiber with a macrolesion. Boxed areas are shown enlarged in panels $\mathbf{b}$ and $\mathbf{c}$. Note the highly extended distance between Z-discs within lesions, and the localization of FLNc between Z-discs. $\mathbf{b}$ Line profile of the boxed area in $\mathbf{a}$ (middle panel) confirms increased signal intensity of FLNc in filamentous appearing structures between Z-discs, while FLNc signals are decreased exactly at the position of the Z-discs. The distance between individual Z-discs increased from 2 to $2.5 \mu \mathrm{m}$ in normal myofibrils to approximately $7.2 \mu \mathrm{m}$ (mean distance: $7.21 \pm 0.73 \mu \mathrm{m}$ ) in lesions. c Line profile of the boxed area in a (lower panel). Note a single normal appearing sarcomere within the macrolesion (asterisk) and the absence of FLNc in this area, whereas the space between the titin signals within the lesion is completely filled with FLNc (arrows). RR90 antibody was used to detect FLNc in a (lower panel), b and c. Polyclonal rabbit antiserum against FLNc d16-20 was used in a (upper panels). Bars: $10 \mu \mathrm{m}(\mathbf{a}), 10 \mu \mathrm{m}(\mathbf{b}), 5 \mu \mathrm{m}(\mathbf{c})$
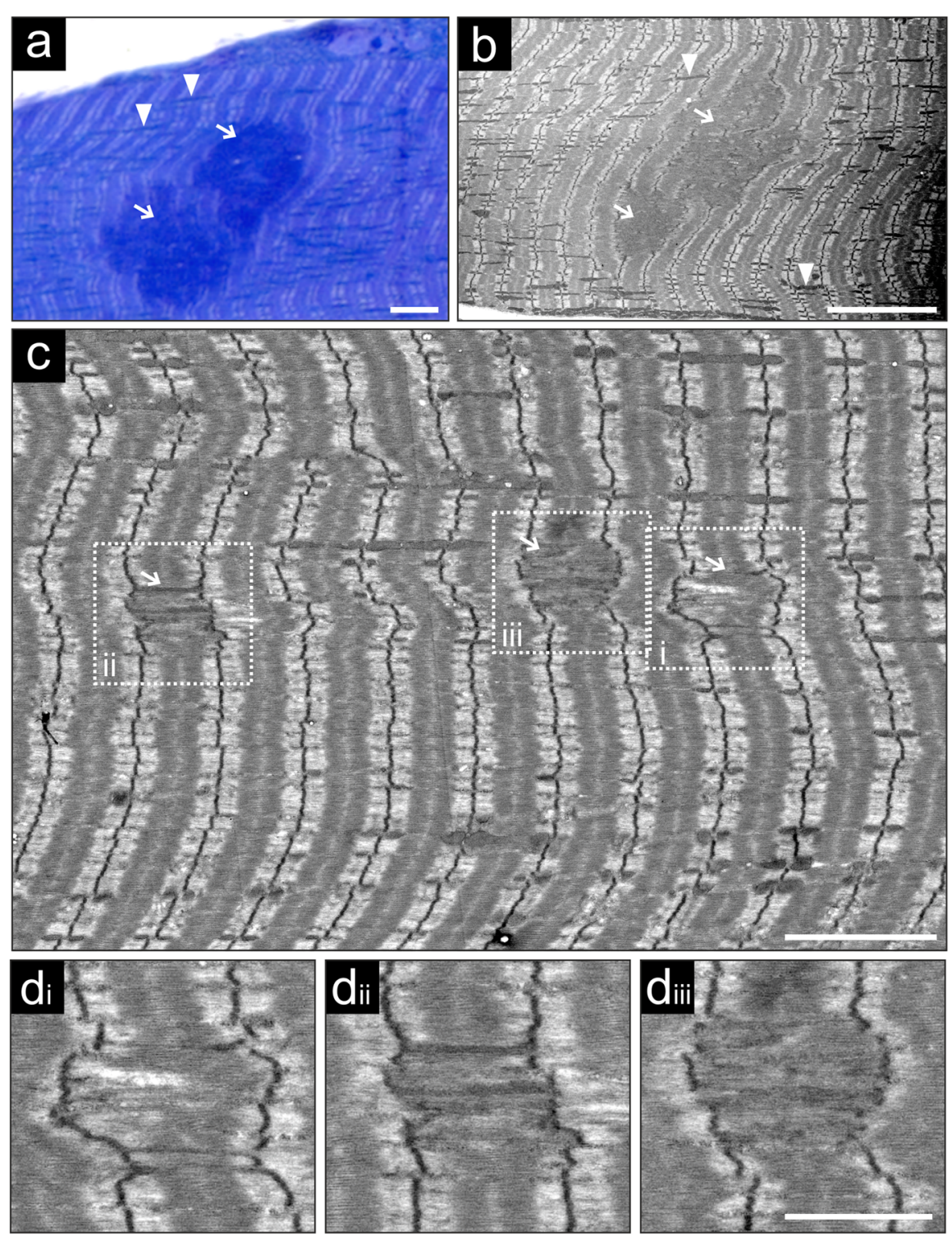

Fig. 6 Soleus muscle of Hom mice at the ultrastructural level. a Longitudinal semithin section of Epon embedded soleus muscle of an adult Hom mouse stained with toluidine blue. Shown is a section with two neighboring darkly stained macrolesions (arrows). Note numerous elongated and swollen mitochondria (arrowheads). b Transmission electron microscopy of an ultrathin section similar to the section shown in A. Depicted are two macrolesions (arrows) surrounded by normally appearing myofibrils. Also, here many elongated and swollen mitochondria are observed (arrowheads). c A section containing three microlesions (arrows). The boxed microlesions are shown enlarged in panels $\mathbf{d}_{i}-\mathbf{d}_{\mathrm{iii}}$. Bars: $5 \mu \mathrm{m}\left(\mathbf{a}_{1}, \mathbf{c}\right)$, $10 \mu \mathrm{m}(\mathbf{b}), 2.5 \mu \mathrm{m}\left(\mathbf{d}_{\mathrm{i}}-\mathbf{d}_{\mathrm{iii}}\right)$ 
macrolesions the normal sarcomere layout of I-bands, A-bands, Z-discs and M-bands was abolished and the space between flanking Z-discs was filled with partially filamentous electron dense material. Notably, these areas were also devoid of mitochondria (Fig. 6b). In addition to macrolesions, many microlesions were observed (Fig. 6c-d), resembling the lesion pathology observed in MFM patient samples. Most microlesions typically extended over a single sarcomere that seemed to gradually lose the normal organization of thin and thick filaments (Fig. 6di), and displayed increasingly disorganized filamentous material. Simultaneously, the distance between the flanking Z-discs increased (Fig. 6dii-diii). Furthermore, we frequently observed rows of elongated and swollen intermyofibrillar mitochondria (Fig. 6a-c) as well as subsarcolemmal autophagic vacuoles (not shown) as previously described for heterozygous p.W2711X animals [11].

\section{Analysis of BAG3 and HSPB7 localization in muscle fibers from WT and Hom mice}

Pathways involved in elimination of misfolded or damaged proteins by autophagy involve BAG3 and HSPB1, HSPB5 and HSPB8, whereas HSPB7 functions independently from BAG3. We analyzed which of these proteins are associated with myofibrillar lesions in WT and Hom muscle fibers. Antibodies against BAG3 as well as HSPB7, brightly stained lesions and Z-discs in muscle fibers from WT mice (Fig. 7a, b, upper panels). By contrast, in the muscle fibers from Hom animals, BAG3 only localized to Z-discs and was not detected in lesions (Fig. 7a, lower panel). The small amounts of HSPB7 that were still expressed in Hom muscle fibers mainly localized to lesions (Fig. 7b, center panel).

\section{Identification of a putative alternative dimerization site in filamin C}

Our previous work demonstrated that the FLNC p.W2710X mutation abolishes the dimerization property of its Ig-like domain 24 [47, 86]. Indications for the presence of an alternative dimerization site within the rod 2 domain of FLNb [70] prompted us to investigate whether also FLNc might have a second dimerization site. We therefore tested the interaction of a construct encompassing FLNc Ig-like domains 15-23 with several other fragments in the yeast-two-hybrid system. Indeed, several fragments interacted (d15-23, d18-21, d19-21), whereas several others (d15-17, d16-19, d21-23) did not. This identified Ig-like domains $19-21$ as the smallest interacting fragment. Notably, deletion of the FLNc-specific insertion in Ig-like domain 20 abolished this interaction (Fig. 8a). To confirm this interaction biochemically, we performed cross-linking assays with FLNc Ig-like domains 18-21, similar to those used to show dimerization of Iglike domain $24[29,47]$. This resulted in higher molecular mass complexes compatible with oligomerization of this FLNc fragment. As noticed in yeast-two-hybrid assays, deletion of the insertion in Ig-like d20 prohibited the interaction. By contrast, a comparable construct comprising FLNa d18-21 did not seem to interact with itself in this assay (Fig. 8b). To confirm this interaction, we also performed pull-down assays in which a GST-FLNc d18-21 fusion protein bound to GSH-beads interacted with FLNc d18-21, but not with FLNc d15-17 (Fig. 8c). This indicates that FLNc lacking Ig-like domain 24, and probably also the p.W2710X mutant variant, might still be able to dimerize or oligomerize.

\section{Discussion}

Until now more than 200 dominant human mutations in the FLNC gene have been identified to be associated with a great variety of myopathies and cardiomyopathies [83]. In addition, very recently a first case of a homozygous, recessive FLNC mutation (NM_001458.4:c.1325C > G; p.P442R) was reported to cause a severe congenital myopathy associated with degenerated myofibrils, advanced sarcomeric lesions and protein aggregates containing FLNc, Xin and XIRP2 in patient muscle fibers [40]. In extension to our previous work on human p.W2710X filaminopathies and patient-mimicking heterozygous Flnc p.W2711X knock-in mice [11, 36-38, 47, 86], we therefore characterized mice homozygous for this mutation. Our hypothesis was that the sole expression of mutant FLNc, containing a truncated, dimerization-incompetent Ig-like domain 24, would enhance pathological processes, thus allowing more precise analysis of the underlying pathomechanisms. One key finding is that, although general striated muscle development and sarcomere formation are not interfered in these mice, even sedentary animals develop a myopathic phenotype in conjunction with a fulminant sarcomeric lesion pathology. By contrast, mice in which the Flnc exons encoding Ig-like domains 21-24 were deleted (Flnc ${ }^{\text {tm1Lmk, }}$ http://www.informatics.jax.org/allele/key/35197) display neonatal lethality caused by defects in primary myogenesis [14], while inducible cardiac-specific ablation of Flnc expression resulted in embryonic death, or death associated with dilated cardiomyopathy in adult mice, respectively [92]. One mechanistic difference explaining the different phenotypes is the fact that the mutations in the latter two mouse models result in highly reduced Flnc mRNA expression, most likely caused by nonsense mediated decay (NMD), and consequently hardly or no detectable levels of mutant FLNc protein $[14,92]$. Therefore, their lethal phenotype has to be attributed to a complete loss of FLNc function. By contrast, the p.W2711X mutation, residing in the last exon, bypasses NMD, resulting in expression of Flnc mRNA at normal levels. This, in turn, implies that the reduced level of mutant protein in our Hom mice is most likely the result of increased FLNc protein 


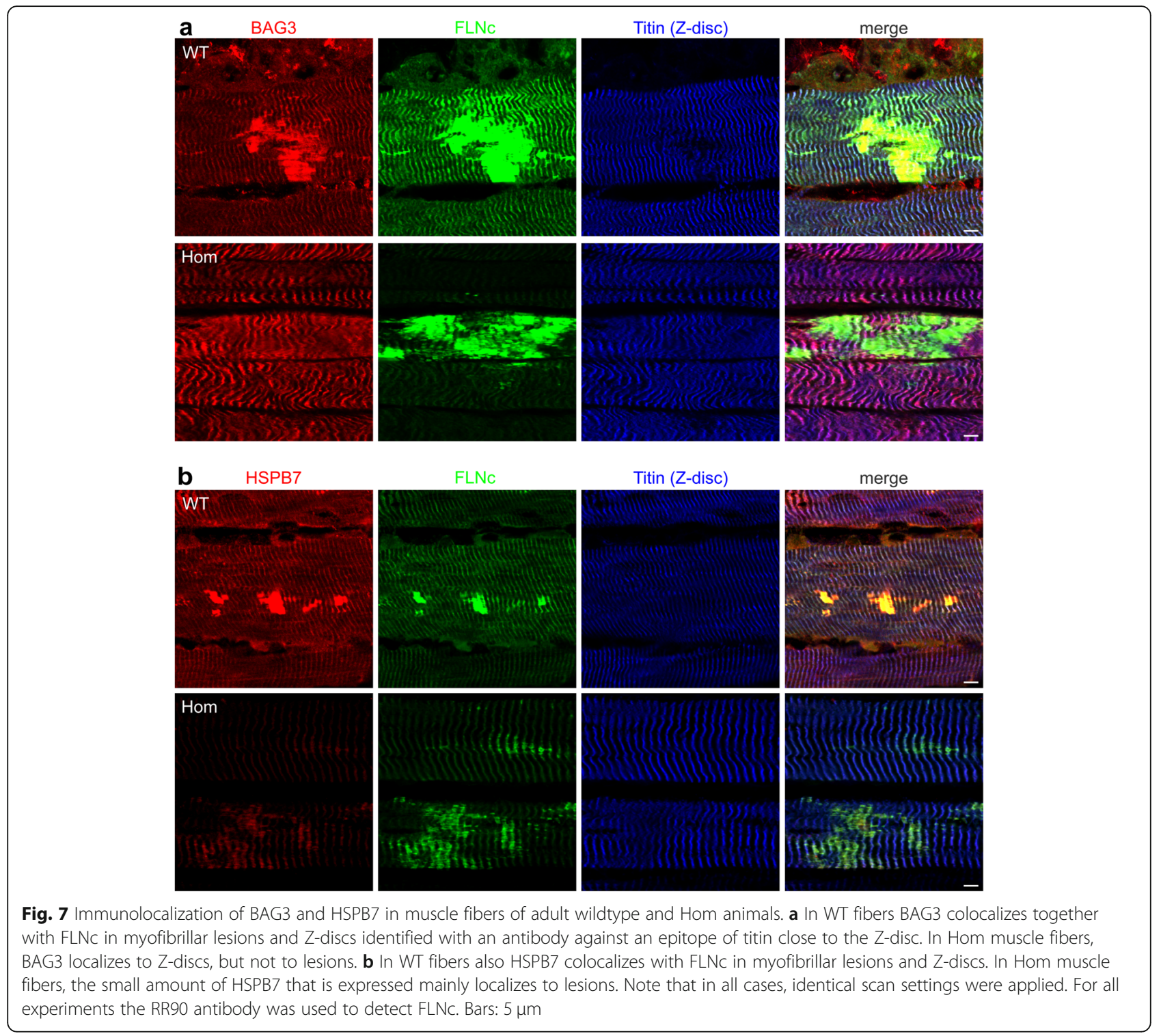

degradation. Notably, FLNC is a dosage-sensitive gene and reduced expression of FLNc causes myopathies [26], indicating that the highly reduced FLNc protein level of our Hom mice is a major mechanism contributing to their phenotype. In this respect it is interesting to note that mass spectrometry analysis of skeletal muscle fibers of heterozygous p.W2711X mice indicated that FLNc levels are reduced to $85.0 \%(p=0.035)$ and $70.1 \%(p=0.0054)$ in type I and type II fibers, respectively (ProteomeXchange submission PXD020097). These differences were previously not detected by non-quantitative western blotting [11].

It is difficult to separate the effect of the reduction of FLNc from that of the expression of mutant FLNc in our mice. Surprisingly few data are available on FLNc expression levels in myopathy patients with an FLNc mutation. In a family with a nonsense mutation (p.F1720LfsX63) that leads to NMD of the mutant mRNA and reduced expression of only wildtype FLNc, the phenotype is compatible with distal myopathy without the typical signs of MFM such as protein aggregation [26]. Patients with an in frame deletion mutation in Ig-like domain 7 (p.V930_ T933del) express normal or even increased levels of FLNc [69], and expression of this unstable and misfolded protein [38] is associated with the formation of aggregates and lesions $[11,38,69]$. A patient with a homozygous p.P442R mutation in Ig-like domain 2 expressed a normal level of unstable FLNc protein and his muscle fibers contained Xin- and FLNc-positive aggregates [40]. This indicates that low FLNc expression levels cause a myopathic phenotype without or with limited aggregates or lesions, whereas the expression of toxic FLNc protein results in lesion and/ or aggregate formation. 


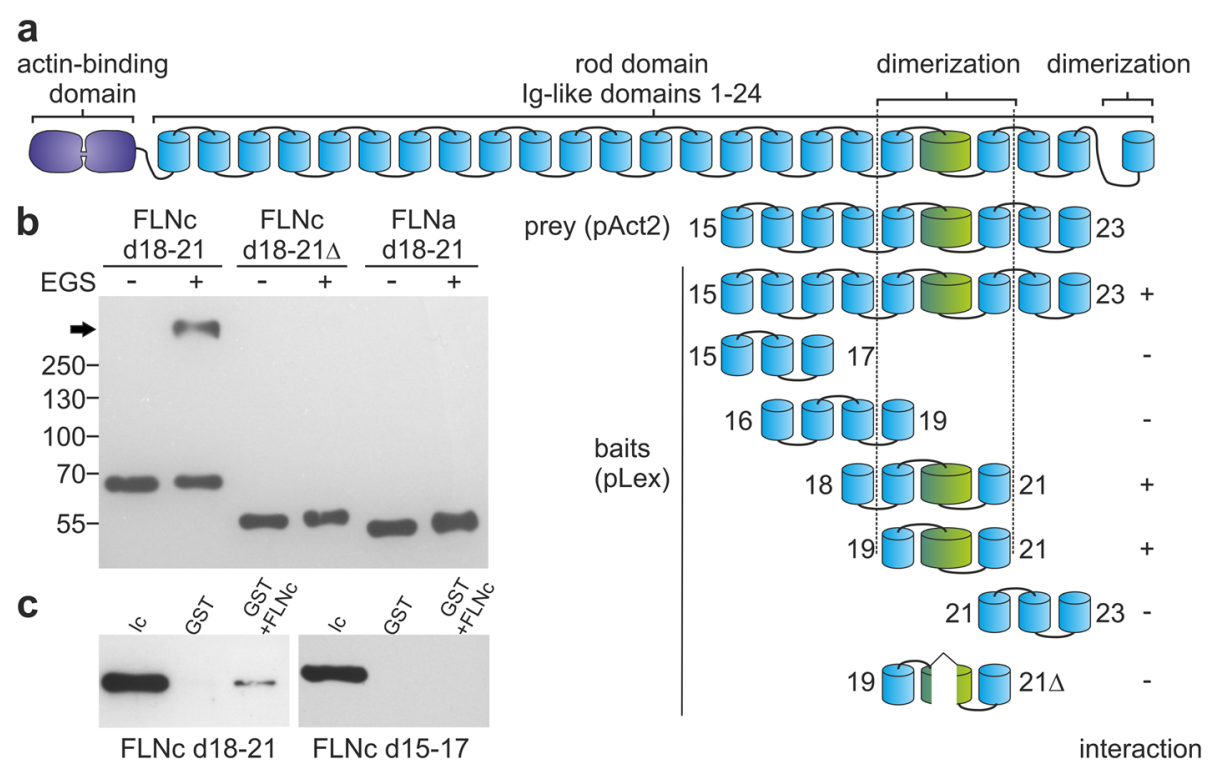

Fig. 8 Analysis of a putative second dimerization region in FLNc. a Yeast-two-hybrid experiments investigating interaction of FLNc lg-like domains 15-23 (prey) with different fragments of FLNc as baits. Interaction was found with d15-23, d18-21 and d19-21 of FLNc. Note that deletion of the FLNc specific insertion in Ig-like domain 20 (d19-21 $\Delta$ ) abolished the interaction. +: interaction; -: no interaction. b FLNc d18-21 with and without $(\Delta)$ insertion in d20, and FLNa d18-21 were incubated in the presence of the cross linker EGS. Separation by PAGE and western blotting with an antibody against the immunotag of the recombinant fragments indicated oligomerization of FLNc d18-21 (arrow) but not of the other constructs. c GST pull down assay indicating that GST-FLNc d19-21 (GST + FLNc) binds T7-tagged FLNc d19-21, but not T7-tagged FLNc d15-17. GST alone does not bind any of the FLNc fragments. Ic: loading control showing the correct size of the FLNc fragments

FLNc is degraded by multiple pathways, including CASA [1, 74], proteasomal degradation [2], and cleavage mediated by calpain 3 [27] or KY protein [3]. Particularly important components for the detection and elimination of damaged FLNc are small heat-shock proteins (sHSPs). The increased expression level of HSPB1, HSPB5 and HSPB8 (and other autophagy-related proteins) especially in type II fibers of our Hom mice (Fig. 2c) points at enhanced autophagic activity. In accordance, in the muscle fibers of MFM-filaminopathy patients, HSPB1 and HSPB5 are strongly upregulated, translocated away from Z-discs [75] and highly concentrated in pathologic aggregates [39]. Notably, these sHSPs interact and cooperate with the co-chaperone and key CASA component BAG3. A major difference between WT, heterozygous p.W2711X and Hom mice is that only in Hom mice BAG3 is not a component of myofibrillar lesions. This implies that an important mechanism contributing to the pathophysiology of the p.W2711X mutation is a lack of full CASA functionality that would be necessary for sufficient repair of the enhanced lesion formation in our Hom mice.

By contrast, the expression level of HSPB7 is highly reduced in the muscle fibers of Hom mice. HSPB7 interacts with the dimerizing domain 24 of FLNc, but not with the p.W2710X variant [31]. HSPB7 was proposed to facilitate early post-damage processing of FLNc and titin [50] and it functions independently from BAG3 [87]. In mice, the complete lack of HSPB7 leads to a progressive myopathy associated with disarray of myofibrils [31]. Cardiacspecific HSPB7 ablation even results in embryonic lethality, concomitant with abnormal actin filament bundles (AABs) within sarcomeres, ultimately resulting in myofibril disorganization [90]. AABs resemble the microlesions we observed in mouse hearts after aortic constriction and in the skeletal muscle fibers of our p.W2711X mice and MFM patients [11, 34]. These AABs lack tropomyosin, troponin, $\mathrm{MyHC}$ and myomesin and are non-contractile [90]. We propose that only in the absence of these proteins, actin cross-linking proteins like FLNc and Xin [57] can bind and stabilize these actin filament bundles. The extremely low expression level of HSPB7 in Hom mice and its inability to interact with mutant FLNc is therefore implied as a major disease mechanism aggravating myofibrillar instability and resulting in increased abundance of micro- and macrolesions. Accordingly, interference with HSPB7 expression in zebrafish and human cardiomyocytes also results in increased load by damaged proteins and stimulation of autophagic pathways [50]. Further studies are necessary to resolve the cause for its strongly reduced expression in our Hom mice, and to elucidate its precise role, both in actin-based assembly and regulation of autophagic pathways [13].

In p.W2711X FLNc only Ig-like domain 24 is misfolded, whereas the other 23 domains remain intact and functional. It is therefore reasonable to assume that most of the functionality of the WT protein is retained, which 
might explain the still relatively subtle phenotype. Heterozygous p.W2711X knock-in mice, which express a mixture of WT and mutant FLNc, revealed an even milder clinical phenotype, with strain-induced sarcomeric macro- and microlesions as most prominent finding [11]. This suggests that mutant FLNc interferes with the maintenance of structural integrity of sarcomeric Zdiscs and mechanical stress resistance. Our present work shows that the sole and reduced expression of mutant FLNc significantly aggravates this phenotype. Human p.W2710X FLNc is prone to aggregate formation in vivo and in vitro $[47,86]$, and its heterozygous expression results in myopathy with lesion and aggregate formation in muscle fibers around the age of 45 years $[36,86]$. The reason why we did not find protein aggregates even in aged Hom mice may simply be that mice do not reach a sufficient age to develop comparable aggregates. We already previously postulated that lesions most likely define preclinical disease stages, preceding the formation of protein aggregates [11]. It was shown that eccentric exercise [21, 53] and electrical pulse stimulation of cultured myotubes [55] leads to increased lesion formation. This would imply that particularly muscles exposed to above average mechanical stress should be particularly affected. In mice, the soleus muscle is the most biomechanically strained muscle during walking, i.e. already under standard housing conditions [30]. Interestingly, FLNc shows its highest expression level in the soleus muscle (Fig. 1a), and here we observed a higher incidence of lesions in comparison to other muscles (results not shown). These findings imply that in humans, muscles which are most strongly strained during walking (like the gastrocnemius, semimembranosus and rectus femoris muscles), should be most strongly affected [30]. Indeed, muscle imaging revealed that the gastrocnemius and semimembranosus muscles are among the most severely affected muscles in human filaminopathy patients [38]. It is thus very likely that strong physical exercise would further aggravate this pathology, an issue important for patient management and to be addressed in future experiments.

Filamins can act as sensors for mechanical stress [17, 41, $62,63,74]$. FLNc is a highly dynamic protein capable of relocating within seconds, e.g. upon inflicting muscle damage by laser-induced microdamage or electrical pacing stimulation $[43,51,55]$. In line with this, in a large variety of human muscle diseases FLNc is dramatically redistributed away from the Z-disc and recruited to subcellular areas with functional imbalances and/or particular mechanical strain such as protein aggregates in myofibrillar myopathies, cores in central and minicore myopathies, subsarcolemmal space in dystrophinopathies, and target lesions in neurogenic atrophies $[8,12,54,68,72,86]$. This is substantiated in our Hom mice that show an almost complete relocalization of (the remaining) mutant FLNc from Zdiscs to myofibrillar lesions and MTJs (see Figs. 3 and 4,
Additional file 1: Figure S4). Notably, this relocalization is not influenced by the lack of the last 16 amino acids. Whereas the complete deletion of Ig-like domain 24 abolishes interaction with sarcoglycans [27], truncation of the C-terminal 16 amino acids alone does not [47]. Likewise, the interaction with Xin, aciculin/PGM5, myotilin, FATZ, myopodin and other ligands that bind FLNc in more amino-terminal regions [20, 45, 51, 81, 82] is not influenced. Accordingly, most of these proteins are found in lesions together with FLNc in the WT as well as in the heterozygous and homozygous mutant situation [19, 34, $43,51,55]$. Furthermore, in a zebrafish model with knockdown of endogenous FLNc, heterologous expression of p.W2710X human FLNc rescues the severe fiber disintegration phenotype, confirming sufficient functionality of the mutant protein [65]. Since also in these fish mutant FLNc is sequestered to attachment sites of myofibrils (comparable to MTJs) and protein aggregates, the authors concluded that the accompanying lack of FLNc (either WT or mutant) in Z-discs is a major cause of myofibrillar instability. The association of FLNc with Xin actinbinding repeat-containing proteins in MTJs and nonstriated parts of myofibrils, including lesions, reduces FLNc dynamics and mobility [43] which might protect the protein in these locations from degradation, and explain its increased localization in these structures.

It is widely accepted that dimerization of filamins is an essential feature of this protein family $[15,52,71]$ and it was surprising that p.W2711X Hom mice do not have a more severe phenotype. This might imply that dimerization is not an essential property of FLNc in striated muscles, or that FLNc does not really cross-link actin filaments in myofibrils. Indeed, an FLNc variant completely lacking Ig-like domain 24 exhibited Z-disc binding but, when compared to the full length variant, higher dynamics in fluorescence recovery after photobleaching (FRAP) experiments [61]. One possible explanation could be an indirect FLNc dimerization achieved by refilins, that contain multiple filamin binding sites [24], or other binding partners capable of forming dimers such as myotilin [66, 85]. An alternative explanation would be the presence of an additional oligomerization site within FLNc as reported for FLNb [70], possibly resulting from intermolecular interactions comparable to the intramolecular interactions reported for domain pair 20-21 in FLNa [28, 41]. Indeed, our yeast two hybrid and in vitro interaction studies were indicative of an additional dimer forming region distinct from Ig-like domain 24.

This leads to the ultimate question what the function of FLNc is in sarcomeric lesions. Considering the fact that FLNc has a plethora of binding partners with highly different functions, we assume that its major task is to orchestrate sarcomere repair by acting as an adaptor protein capable of recruiting both signaling and structural components. Our immunostainings of macrolesions imply 
that locally titin molecules are maximally stretched to a Zdisc epitope spacing of approximately $7.2 \mu \mathrm{m}$. This distance is compatible with the length of two titin molecules stretched to their maximal extension $[7,35]$. In these regions FLNc is relocated from Z-discs to the space between these far distant titin epitopes (see Fig. 4), where a noncontractile and elastic filamentous scaffold is formed to reduce the mechanical stress in order to prevent further damage and to retain the continuity of the contractile apparatus $[43,55]$. As a protein with many binding partners, FLNc can then recruit other proteins necessary for repair of the damaged sarcomeres [43].

Further studies addressing the sequential molecular events and the time course of lesion formation and repair, and the involvement of autophagy in this context are needed to understand this crucial process in detail.

\section{Conclusions}

Homozygosity for the MFM-associated p.W2711X mutation in Flnc in mice leads to increased lesion formation in skeletal muscle fibers even without additional exposure to strenuous mechanical stress. Our analyses revealed the following major mechanisms contributing to the pathophysiological condition of filaminopathy:

(1) although Flnc mRNA expression levels are not reduced, muscle fibers from Hom mice express highly reduced levels of FLNc protein.

(2) mutant FLNc relocates from Z-discs to particularly mechanically strained parts of muscle cells;

(3) muscle fibers from Hom mice display dysregulated sHSP expression: while HSPB7 is lacking, HSPB1, HSPB5 and HSPB8 are upregulated; the absence of HSPB7 may directly contribute to sarcomere disorganization;

(4) dysregulation of CASA is indicated by the absence of BAG3 from lesions and concomitant upregulation of the adapter protein SYNPO2; consequently, clearance of mechanically damaged proteins from lesions is compromised.

Homozygous FLNC p.W2711X mice will be an excellent tool for investigations aiming at understanding sarcomeric lesion formation, and to find possibilities to prevent muscle weakness by lesion formation or to find methods to enhance lesion repair.

\section{Supplementary information}

Supplementary information accompanies this paper at https://doi.org/10. 1186/s40478-020-01001-9.

Additional file 1: Figure S1. Histochemistry performed on 8-month-old wild type (WT) and mutant (Hom) mouse soleus muscles. Figure S2. Analysis of fibre types in soleus muscle of adult and aged wildtype and
Hom mice. Figure S3. Distribution of filamin C and vinculin in myotendinous junctions. Table S1. Specification of primary antibodies used in this study. Table S2. Sequence of oligonucleotides used for quantitative real time PCR.

\section{Abbreviations}

BAG3: Bcl2-associated athanogene 3 protein; BSA: Bovine serum albumin; CLB: Cross-link buffer; COX: Cytochrome oxidase; EDL: Extensor digitorum longus; EGFP: Enhanced green fluorescent protein; FATZ: Filamin-, actinin-, and telethonin-binding protein of the Z-disc; FDR: False discovery rate; FLN: Filamin; GAPDH: Glyceraldehyde-3-phosphate dehydrogenase; GST: Glutathione S-transferase; HE: Haematoxylin and eosin; HSPB7: Heat shock protein beta-7; Ig-like: immunoglobulin-like; LMD: Laser microdissection; MFM: Myofibrillar myopathy; MHC: Myosin heavy chain; MS: Mass spectrometry; MTJ: Myotendinous junction; NMD: Nonsense mediated decay; PAGE: Polyacrylamide gel electrophoresis;

PBS(T): Phosphate-buffered saline (with Tween 20);

PGM: Phosphoglucomutase; PSMs: Peptide spectrum matches;

PVDF: Polyvinylidenfluoride; RT-qPCR: Reverse transcription quantitative polymerase chain reaction; SDH: Succinate dehydrogenase; TA: Tibialis anterior; TBS(T): Tris-buffered saline (with Tween 20); WT: Wildtype

\section{Acknowledgements}

The authors are very grateful to Dr. Michael Welter, Mathematics Institute, University of Bonn for his invaluable advice on statistical analyses. They also would like to thank Karin Bois, Corina Mirschkorsch, Babette Bockmühl and Ute Kukulies for excellent technical assistance.

\section{Authors' contributions}

DOF and RS conceived and designed the study; JS, ZO, FC, BE, LH, JU performed experiments; CSC contributed materials; JS, ZO, JU KM, GK analyzed data; PFMvdV, CSC, AU, WAL provided scientific input; PFMvdV and DOF wrote the manuscript with input from all authors. All authors were involved in revising the manuscript. All authors read and approved the final manuscript.

\section{Funding}

This work was supported by the Else Kröner-Fresenius-Stiftung (Grant Number: 2011A46 to DOF and RS), the Multilocation DFG-Research Units FOR1352 and FOR2743 from the German Research Foundation (Grant Numbers: Fu 339/8-2 and Fu 339/11-1 to DOF), the Association Française contre les Myopathies (FC), Protein research Unit Ruhr within Europe (PURE) a project of Northrhine-Westphalia (KM, JU, BE), and de. NBI (FKZ 031 A 534A; a project of the German Federal Ministry of Education and Research, BMBF) $(K M, J U)$. Open access funding provided by Projekt DEAL.

\section{Availability of data and materials}

The datasets used and/or analyzed during the current study available from the corresponding author on reasonable request. Mass spectrometry proteomics data have been deposited to the ProteomeXchange Consortium via the PRIDE partner repository with the dataset identifier PXD020097.

Ethics approval and consent to participate

All investigations were approved by the governmental office for animal care (reference number 84-02.05.40.14.057).

\section{Consent for publication}

Not applicable.

\section{Competing interests}

The authors declare that they have no conflict of interest.

\section{Author details}

${ }^{1}$ Institute for Cell Biology, Department of Molecular Cell Biology, University of Bonn, Ulrich-Haberland-Str. 61a, 53121 Bonn, Germany. ${ }^{2}$ Present address: Institute of Virology, University Hospital Giessen and Marburg, Marburg, Germany. ${ }^{3}$ Institute of Neuropathology, University Hospital Erlangen, Friedrich-Alexander University Erlangen-Nürnberg, Schwabachanlage 6, 91054 Erlangen, Germany. ${ }^{4}$ present address: CureVac AG, Tübingen, Germany. ${ }^{5}$ Medizinisches Proteom-Center, Ruhr-University Bochum, Bochum, Germany. ${ }^{6}$ Institute of Physiology II, University of Münster, Münster, Germany. ${ }^{7}$ Center 
for Biochemistry, Institute of Biochemistry I, Medical Faculty, University of Cologne, Cologne, Germany. ${ }^{8}$ Center for Physiology and Pathophysiology, Institute of Vegetative Physiology, Medical Faculty, University of Cologne, Cologne, Germany. ${ }^{9}$ Institute of Aerospace Medicine, German Aerospace Center (DLR), Cologne, Germany.

Received: 21 April 2020 Accepted: 22 July 2020

Published online: 04 September 2020

\section{References}

1. Arndt V, Dick N, Tawo R, Dreiseidler M, Wenzel D, Hesse M et al (2010) Chaperone-assisted selective autophagy is essential for muscle maintenance. Curr Biol 20:143-148

2. Baldassarre M, Razinia Z, Burande CF, Lamsoul I, Lutz PG, Calderwood DA (2009) Filamins regulate cell spreading and initiation of cell migration. PLoS One 4:e7830

3. Beatham J, Romero R, Townsend SK, Hacker T, van der Ven PFM, Blanco G (2004) Filamin C interacts with the muscular dystrophy KY protein and is abnormally distributed in mouse KY deficient muscle fibres. Hum Mol Genet 13:2863-2874

4. Begay RL, Tharp CA, Martin A, Graw SL, Sinagra G, Miani D et al (2016) FLNC gene splice mutations cause dilated cardiomyopathy. JACC Basic Transl Sci 1:344-359

5. Begay RL, Graw SL, Sinagra G, Asimaki A, Rowland TJ, Slavov DB et al (2018) Filamin $C$ truncation mutations are associated with arrhythmogenic dilated cardiomyopathy and changes in the cell-cell adhesion structures. JACC Clin Electrophysiol 4:504-514

6. Berthold MR, Cebron N, Dill F, Gabriel TR, Kötter T, Meinl T et al (2008) KNIM E. The Konstanz information miner. In: Preisach C, Burkhardt H, SchmidtThieme $L$ et al (eds) Data Analysis, Machine Learning and Applicatieons. Springer, Berlin Heidelberg, pp 319-326

7. Bianco P, Mártonfalvi Z, Naftz K, Kőszegi D, Kellermayer M (2015) Titin domains progressively unfolded by force are homogenously distributed along the molecule. Biophys J 109:340-345

8. Bönnemann CG, Thompson TG, van der Ven PFM, Goebel HH, Warlo I, Vollmers B et al (2003) Filamin C accumulation is a strong but nonspecific immunohistochemical marker of core formation in muscle. J Neurol Sci 206:71-78

9. Brodehl A, Ferrier RA, Hamilton SJ, Greenway SC, Brundler M-A, Yu W et al (2015) Mutations in FLNC are associated with familial restrictive cardiomyopathy. Hum Mutat 37:269-279

10. Chen J, Shinde S, Koch M-H, Eisenacher M, Galozzi S, Lerari T et al (2015) Low-bias phosphopeptide enrichment from scarce samples using plastic antibodies. Sci Rep 5:11438

11. Chevessier F, Schuld J, Orfanos Z, Plank A-C, Wolf L, Maerkens A et al (2015) Myofibrillar instability exacerbated by acute exercise in filaminopathy. Hum Mol Genet 24:7207-7220

12. Claeys KG, van der Ven PFM, Behin A, Stojkovic T, Eymard B, Dubourg O et al (2009) Differential involvement of sarcomeric proteins in myofibrillar myopathies: a morphological and immunohistochemical study. Acta Neuropathol 117:293-307

13. Collier MP, Benesch JLP (2020) Small heat-shock proteins and their role in mechanical stress. Cell Stress Chaperones 25:601-613

14. Dalkilic I, Schienda J, Thompson TG, Kunkel LM (2006) Loss of FilaminC (FLNC) results in severe defects in myogenesis and myotube structure. $\mathrm{Mo}$ Cell Biol 26:6522-6534

15. Djinović-Carugo K, Carugo O (2010) Structural portrait of filamin interaction mechanisms. Curr Protein Pept Sci 11:639-650

16. Duff RM, Tay V, Hackman P, Ravenscroft G, McLean C, Kennedy P et al (2011) Mutations in the $\mathrm{N}$-terminal actin-binding domain of filamin $C$ cause a distal myopathy. Am J Hum Genet 88:729-740

17. Ehrlicher AJ, Nakamura F, Hartwig JH, Weitz DA, Stossel TP (2011) Mechanical strain in actin networks regulates FilGAP and integrin binding to filamin a. Nature 478:260-263

18. Ehsan M, Jiang $\mathrm{H}$, Thomson $\mathrm{KL}$, Gehmlich K (2017) When signalling goes wrong. Pathogenic variants in structural and signalling proteins causing cardiomyopathies. J Muscle Res Cell Motil 38:303-316

19. Eulitz S, Sauer F, Pelissier MC, Boisguerin P, Molt S, Schuld J et al (2013) Identification of Xin-repeat proteins as novel ligands of the SH3 domains of nebulin and nebulette and analysis of their interaction during myofibril formation and remodeling. Mol Biol Cell 24:3215-3226
20. Faulkner G, Pallavicini A, Comelli A, Salamon M, Bortoletto G, levolella C et al (2000) FATZ: a filamin-, actinin-, and telethonin-binding protein of the Z-disk of skeletal muscle. J Biol Chem 275:41234-41242

21. Fridén J, Sjöström M, Ekblom B (1983) Myofibrillar damage following intense eccentric exercise in man. Int J Sports Med 4:170-176

22. Fürst DO, Osborn M, Nave R, Weber K (1988) The organization of titin filaments in the half-sarcomere revealed by monoclonal antibodies in immunoelectron microscopy: a map of ten nonrepetitive epitopes starting at the $\mathbf{Z}$ line extends close to the $\mathrm{M}$ line. J Cell Biol 106:1563-1572

23. Fürst DO, Goldfarb LG, Kley RA, Vorgerd M, Olivé M, van der Ven PFM (2013) Filamin C-related myopathies: pathology and mechanisms. Acta Neuropathol 125:33-46

24. Gay O, Gilquin B, Nakamura F, Jenkins ZA, McCartney R, Krakow D et al (2011) RefilinB (FAM101B) targets filamin a to organize perinuclear actin networks and regulates nuclear shape. Proc Natl Acad Sci U S A 108:1146411469

25. Gómez J, Lorca R, Reguero JR, Morís C, Martín M, Tranche S et al (2017) Screening of the filamin $C$ gene in a large cohort of hypertrophic cardiomyopathy patients. Circ Cardiovasc Genet 10:e001584

26. Guergueltcheva V, Peeters K, Baets J, Ceuterick-de Groote C, Martin JJ, Suls A et al (2011) Distal myopathy with upper limb predominance caused by filamin C haploinsufficiency. Neurology 77:2105-2114

27. Guyon JR, Kudryashova E, Potts A, Dalkilic I, Brosius MA, Thompson TG et al (2003) Calpain 3 cleaves filamin $C$ and regulates its ability to interact with $\gamma$ and $\delta$-sarcoglycans. Muscle Nerve 28:472-483

28. Heikkinen OK, Ruskamo S, Konarev PV, Svergun DI, livanainen T, Heikkinen SM et al (2009) Atomic structures of two novel immunoglobulin-like domain pairs in the actin cross-linking protein filamin. J Biol Chem 284: 25450-25458

29. Himmel M, van der Ven PFM, Stöcklein W, Fürst DO (2003) The limits of promiscuity: isoform-specific dimerization of filamins. Biochemistry 42:430-439

30. Hu X, Charles JP, Akay T, Hutchinson JR, Blemker SS (2017) Are mice good models for human neuromuscular disease? Comparing muscle excursions in walking between mice and humans. Skelet Muscle 7:26

31. Juo L-Y, Liao W-C, Shih Y-L, Yang B-Y, Liu A-B, Yan Y-T (2016) HSPB7 interacts with dimerized FLNC and its absence results in progressive myopathy in skeletal muscles. J Cell Sci 129:1661-1670

32. Karmouch J, Protonotarios A, Syrris P (2018) Genetic basis of arrhythmogenic cardiomyopathy. Curr Opin Cardiol 33:276-281

33. Karnovsky MJ (1965) A formaldehyde-glutaraldehyde fixative of high osmolality for use in electron microscopy. J Cell Biol 27:137A-138A

34. Kebir S, Orfanos Z, Schuld J, Linhart M, Lamberz C, van der Ven PFM et al (2016) Sarcomeric lesions and remodeling proximal to intercalated disks in overload-induced cardiac hypertrophy. Exp Cell Res 348:95-105

35. Kellermayer M, Smith SB, Bustamante C, Granzier HL (1998) Complete unfolding of the titin molecule under external force. J Struct Biol 122:197-205

36. Kley RA, Hellenbroich Y, van der Ven PFM, Fürst DO, Huebner A, Bruchertseifer $V$ et al (2007) Clinical and morphological phenotype of the filamin myopathy: a study of 31 German patients. Brain 130:3250-3264

37. Kley RA, van der Ven PFM, Olivé M, Höhfeld J, Goldfarb LG, Fürst DO et al (2012) Impairment of protein degradation in myofibrillar myopathy caused by FLNC/filamin C mutations. Autophagy 9:422-423

38. Kley RA, Serdaroglu-Oflazer P, Leber Y, Odgerel Z, van der Ven PFM, Olivé M et al (2012) Pathophysiology of protein aggregation and extended phenotyping in filaminopathy. Brain 135:2642-2660

39. Kley RA, Maerkens A, Leber $Y$, Theis $V$, Schreiner $A$, van der Ven PFM et al (2013) A combined laser microdissection and mass spectrometry approach reveals new disease relevant proteins accumulating in aggregates of filaminopathy patients. Mol Cell Proteomics 12:215-227

40. Kölbel H, Roos A, van der Ven PFM, Evangelista T, Nolte K, Johnson $\mathrm{K}$ et al (2020) First clinical and myopathological description of a myofibrillar myopathy with congenital onset and homozygous mutation in FLNC. Hum Mutat https:// doi.org/10.1002/humu.24062. Online ahead of print

41. Lad Y, Kiema T, Jiang P, Pentikainen OT, Coles CH, Campbell ID et al (2007) Structure of three tandem filamin domains reveals auto-inhibition of ligand binding. EMBO J 26:3993-4004

42. Laemmli UK (1970) Cleavage of structural proteins during the assembly of the head of bacteriophage T4. Nature 227:680-685

43. Leber $Y$, Ruparelia AA, Kirfel G, van der Ven PFM, Hoffmann B, Merkel R et al (2016) Filamin C is a highly dynamic protein associated with fast repair of myofibrillar microdamage. Hum Mol Genet 25:2776-2788 
44. Lee H-CH, Wong S, Sheng B, Pan N-YK, Leung Y-KF, Lau K-KD et al (2020) Clinical and pathological characterization of FLNC-related myofibrillar myopathy caused by founder variant c.8129G A in Hong Kong Chinese. Clin Genet 97:747-757

45. Linnemann A, van der Ven PFM, Vakeel P, Albinus B, Simonis D, Bendas G et al (2010) The sarcomeric Z-disc component myopodin is a multiadapte protein that interacts with filamin and a-actinin. Eur J Cell Biol 89:681-692

46. Livak KJ, Schmittgen TD (2001) Analysis of relative gene expression data using real-time quantitative PCR and the 2(-Delta Delta $C(T))$ method. Methods 25:402-408

47. Löwe T, Kley RA, van der Ven PFM, Himmel M, Huebner A, Vorgerd M et al (2007) The pathomechanism of filaminopathy: altered biochemical properties explain the cellular phenotype of a protein aggregation myopathy. Hum Mol Genet 16:1351-1358

48. Maerkens A, Kley RA, Olivé $M$, Theis $V$, van der Ven PFM, Reimann J et al (2013) Differential proteomic analysis of abnormal intramyoplasmic aggregates in desminopathy. J Proteome 90:14-27

49. Maerkens A, Olivé M, Schreiner A, Feldkirchner S, Schessl J, Uszkoreit J et al (2016) New insights into the protein aggregation pathology in myotilinopathy by combined proteomic and immunolocalization analyses. Acta Neuropathol Comm 4:549

50. Mercer EJ, Lin Y-F, Cohen-Gould L, Evans T (2018) Hspb7 is a cardioprotective chaperone facilitating sarcomeric proteostasis. Dev Biol 435:41-55

51. Molt S, Bührdel JB, Yakovlev S, Schein P, Orfanos Z, Kirfel G et al (2014) Aciculin interacts with filamin $C$ and Xin and is essential for myofibril assembly, remodeling and maintenance. J Cell Sci 127:3578-3592

52. Nakamura F, Stossel TP, Hartwig JH (2011) The filamins: organizers of cell structure and function. Cell Adhes Migr 5:160-169

53. Newham DJ, McPhail G, Mills KR, Edwards RH (1983) Ultrastructural changes after concentric and eccentric contractions of human muscle. J Neurol Sci 61:109-122

54. Nilsson MI, Nissar AA, Al-Sajee D, Tarnopolsky MA, Parise G, Lach B et al (2013) Xin is a marker of skeletal muscle damage severity in myopathies. Am J Pathol 183:1703-1709

55. Orfanos Z, Gödderz MPO, Soroka E, Gödderz T, Rumyantseva A, van der Ven PFM et al (2016) Breaking sarcomeres by in vitro exercise. Sci Rep 6:19614

56. Ortiz-Genga MF, Cuenca S, Dal Ferro M, Zorio E, Salgado-Aranda R, Climent V et al (2016) Truncating FLNC mutations are associated with high-risk dilated and arrhythmogenic cardiomyopathies. J Am Coll Cardiol 68:2440-2451

57. Pacholsky D, Vakeel P, Himmel M, Löwe T, Stradal T, Rottner K et al (2004) Xin repeats define a novel actin-binding motif. J Cell Sci 117:5257-5268

58. Perez-Riverol Y, Csordas A, Bai J, Bernal-Llinares M, Hewapathirana S, Kundu DJ et al (2019) The PRIDE database and related tools and resources in 2019: improving support for quantification data. Nucleic Acids Res 47:D442-D450

59. Perkins DN, Pappin DJ, Creasy DM, Cottrell JS (1999) Probability-based protein identification by searching sequence databases using mass spectrometry data. Electrophoresis 20:3551-3567

60. Reidegeld KA, Eisenacher M, Kohl M, Chamrad D, Körting G, Blüggel M et al (2008) An easy-to-use decoy database builder software tool, implementing different decoy strategies for false discovery rate calculation in automated MS/MS protein identifications. Proteomics 8:1129-1137

61. Reimann L, Wiese H, Leber Y, Schwäble AN, Fricke AL, Rohland A et al (2017) Myofibrillar Z-discs are a protein phosphorylation hot spot with protein kinase C (PKCa) modulating protein dynamics. Mol Cell Proteomics 16:346-367

62. Rognoni L, Stigler J, Pelz B, Ylänne J, Rief M (2012) Dynamic force sensing of filamin revealed in single-molecule experiments. Proc Natl Acad Sci U S A 109:19679-19684

63. Rognoni L, Möst T, Žoldák G, Rief M (2014) Force-dependent isomerization kinetics of a highly conserved proline switch modulates the mechanosensing region of filamin. Proc Natl Acad Sci U S A 111:5568-5573

64. Ruparelia AA, Zhao M, Currie PD, Bryson-Richardson RJ (2012) Characterization and investigation of zebrafish models of filamin-related myofibrillar myopathy. Hum Mol Genet 21:4073-4083

65. Ruparelia AA, Oorschot V, Ramm G, Bryson-Richardson RJ (2016) FLNC myofibrillar myopathy results from impaired autophagy and protein insufficiency. Hum Mol Genet 25:2131-2142

66. Salmikangas $P$, van der Ven PFM, Lalowski M, Taivainen A, Zhao F, Suila $H$ et al (2003) Myotilin, the limb-girdle muscular dystrophy 1A (LGMD1A) protein, cross-links actin filaments and controls sarcomere assembly. Hum Mol Genet 12:189-203

67. Schröder R, Schoser B (2009) Myofibrillar myopathies: a clinical and myopathological guide. Brain Pathol 19:483-492
68. Sewry CA (2000) Immunocytochemical analysis of human muscular dystrophy. Microsc Res Tech 48:142-154

69. Shatunov A, Olivé M, Odgerel Z, Stadelmann-Nessler C, Irlbacher K, van Landeghem F et al (2009) In-frame deletion in the seventh immunoglobulin-like repeat of filamin $C$ in a family with myofibrillar myopathy. Eur J Hum Genet 17:656-663

70. Sheen VL, Feng Y, Graham D, Takafuta T, Shapiro SS, Walsh CA (2002) Filamin a and Filamin $B$ are co-expressed within neurons during periods of neuronal migration and can physically interact. Hum Mol Genet 11:2845-2854

71. Stossel TP, Condeelis J, Cooley L, Hartwig JH, Noegel A, Schleicher M et al (2001) Filamins as integrators of cell mechanics and signalling. Nat Rev Mol Cell Biol 2:138-145

72. Thompson TG, Chan YM, Hack AA, Brosius M, Rajala M, Lidov HG et al (2000) Filamin 2 (FLN2). A muscle-specific sarcoglycan interacting protein. J Cell Biol 148:115-126

73. Turewicz M, Kohl M, Ahrens M, Mayer G, Uszkoreit J, Naboulsi W et al (2017) Biolnfra.Prot. A comprehensive proteomics workflow including data standardization, protein inference, expression analysis and data publication. J Biotechnol 261:116-125

74. Ulbricht A, Eppler FJ, Tapia VE, van der Ven PFM, Hampe N, Hersch N et al (2013) Cellular mechanotransduction relies on tension-induced and chaperone-assisted autophagy. Curr Biol 23:430-435

75. Unger A, Beckendorf L, Böhme P, Kley RA, von Frieling-Salewsky M, Lochmüller $\mathrm{H}$ et al (2017) Translocation of molecular chaperones to the titin springs is common in skeletal myopathy patients and affects sarcomere function. Acta Neuropathol Comm 5:72

76. UniProt Consortium T (2018) UniProt. The universal protein knowledgebase. Nucleic Acids Res 46:2699

77. Uszkoreit J, Maerkens A, Perez-Riverol Y, Meyer HE, Marcus K, Stephan C et al (2015) PIA. An intuitive protein inference engine with a web-based user interface. J Proteome Res 14:2988-2997

78. Valdés-Mas R, Gutiérrez-Fernández A, Gómez J, Coto E, Astudillo A, Puente DA et al (2014) Mutations in filamin C cause a new form of familial hypertrophic cardiomyopathy. Nat Commun 5:5326

79. van der Flier A, Sonnenberg A (2001) Structural and functional aspects of filamins. Biochim Biophys Acta 1538:99-117

80. van der Ven PFM, Obermann WMJ, Lemke B, Gautel M, Weber K, Fürst DO (2000) Characterization of muscle filamin isoforms suggests a possible role of $\gamma$-filamin/ ABP-L in sarcomeric Z-disc formation. Cell Motil Cytoskeleton 45:149-162

81. van der Ven PFM, Wiesner S, Salmikangas P, Auerbach D, Himmel M, Kempa $S$ et al (2000) Indications for a novel muscular dystrophy pathway. $\gamma$-filamin, the muscle-specific filamin isoform, interacts with myotilin. J Cell Biol 151:235-248

82. van der Ven PFM, Ehler E, Vakeel P, Eulitz S, Schenk JA, Milting $H$ et al (2006) Unusual splicing events result in distinct Xin isoforms that associate differentially with filamin C and Mena/VASP. Exp Cell Res 312:2154-2167

83. Verdonschot JAJ, Vanhoutte EK, Claes GRF, Helderman-van den Enden ATJM, Hoeijmakers JGJ, Hellebrekers DMEl et al (2020) A mutation update for the FLNC gene in myopathies and cardiomyopathies. Hum Mutat 41:1091-1111

84. Vinkemeier U, Obermann W, Weber K, Fürst DO (1993) The globular head domain of titin extends into the center of the sarcomeric $M$ band. cDNA cloning, epitope mapping and immunoelectron microscopy of two titinassociated proteins. J Cell Sci 106:319-330

85. von Nandelstadh P, Grönholm M, Moza M, Lamberg A, Savilahti H, Carpén O (2005) Actin-organising properties of the muscular dystrophy protein myotilin. Exp Cell Res 310:131-139

86. Vorgerd M, van der Ven PFM, Bruchertseifer V, Löwe T, Kley RA, Schröder R et al (2005) A mutation in the dimerization domain of filamin c causes a novel type of autosomal dominant myofibrillar myopathy. Am J Hum Genet 77:297-304

87. Vos MJ, Zijlstra MP, Kanon B, van Waarde-Verhagen MAWH, Brunt ERP, Oosterveld-Hut HMJ et al (2010) HSPB7 is the most potent polyQ aggregation suppressor within the HSPB family of molecular chaperones. Hum Mol Genet 19:4677-4693

88. Wehland J, Willingham MC, Sandoval IV (1983) A rat monoclonal antibody reacting specifically with the tyrosylated form of alpha-tubulin. I. Biochemical characterization, effects on microtubule polymerization in vitro, and microtubule polymerization and organization in vivo. J Cell Biol 97:1467-1475

89. Winter L, Wittig I, Peeva V, Eggers B, Heidler J, Chevessier F et al (2016) Mutant desmin substantially perturbs mitochondrial morphology, function and maintenance in skeletal muscle tissue. Acta Neuropathol $132: 453-473$ 
90. Wu T, Mu Y, Bogomolovas J, Fang X, Veevers J, Nowak RB et al (2017) HSPB7 is indispensable for heart development by modulating actin filament assembly. Proc Natl Acad Sci U S A 114:11956-11961

91. Zhou X, Boren J, Akyurek LM (2007) Filamins in cardiovascular development. Trends Cardiovasc Med 17:222-229

92. Zhou Y, Chen Z, Zhang L, Zhu M, Tan C, Zhou X et al (2020) Loss of filamin $C$ is catastrophic for heart function. Circulation 141:869-871

\section{Publisher's Note}

Springer Nature remains neutral with regard to jurisdictional claims in published maps and institutional affiliations.

Ready to submit your research? Choose BMC and benefit from:

- fast, convenient online submission

- thorough peer review by experienced researchers in your field

- rapid publication on acceptance

- support for research data, including large and complex data types

- gold Open Access which fosters wider collaboration and increased citations

- maximum visibility for your research: over $100 \mathrm{M}$ website views per year

At BMC, research is always in progress.

Learn more biomedcentral.com/submissions 